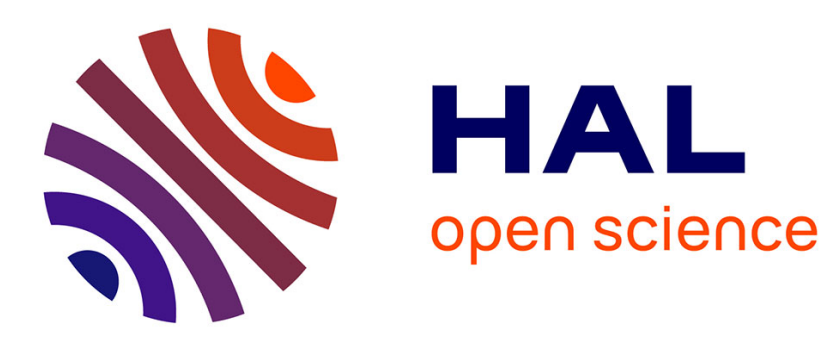

\title{
Expérience carcérale et exercice de la paternité : le point de vue de pères détenus
}

\author{
Marine Quennehen
}

\section{To cite this version:}

Marine Quennehen. Expérience carcérale et exercice de la paternité : le point de vue de pères détenus. Champ Pénal, 2019, 16, 10.4000/champpenal.10397 . hal-02140356

\section{HAL Id: hal-02140356 \\ https://hal.science/hal-02140356}

Submitted on 27 May 2019

HAL is a multi-disciplinary open access archive for the deposit and dissemination of scientific research documents, whether they are published or not. The documents may come from teaching and research institutions in France or abroad, or from public or private research centers.
L'archive ouverte pluridisciplinaire HAL, est destinée au dépôt et à la diffusion de documents scientifiques de niveau recherche, publiés ou non, émanant des établissements d'enseignement et de recherche français ou étrangers, des laboratoires publics ou privés. 


\section{Expérience carcérale et exercice de la paternité : le point de vue de pères détenus}

Prison experience and paternity: the point of view of imprisoned fathers Marine Quennehen

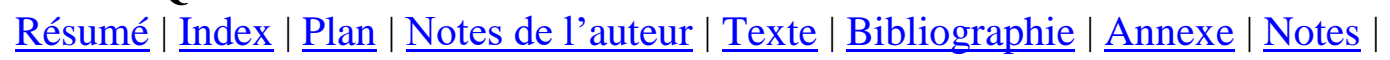

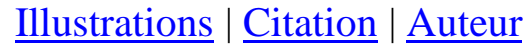

\section{Résumés}

\section{$\underline{\text { Français English }}$}

Comment l'incarcération affecte-t-elle le rôle paternel ? La prison ne peut être considérée comme un isolat social. Sans modifier complètement la manière dont les individus vivent et se représentent leur paternité, sans que le contexte carcéral la conditionne de façon déterministe, elle y subit nécessairement des transformations : ce milieu produit des conditions spécifiques d'actualisation de ce rôle et des caractéristiques nouvelles. La notion de « filet » proposée par Norbert Elias (1997) nous permet de concevoir la paternité comme un filet tissé de multiples fils reliés entre eux. Dès lors, plutôt que d'expliquer la paternité à partir d'un seul fil (le lien père-enfant), c'est à partir de tous ces fils (histoire familiale, conjugale, facteurs socioculturels, etc.) et de leurs connexions que nous en proposons une approche pluridimensionnelle.

Pour appréhender de façon cohérente ces différentes dimensions de la paternité, nous distinguons et lions trois niveaux : une analyse relationnelle de la paternité en contexte d'incarcération saisie par les pratiques concrètes des pères détenus et de leur entourage, une approche biographique replaçant le présent dans l'histoire familiale des pères rencontrés et enfin par une analyse « compréhensive » des normes (au sens de Max Weber), s'efforçant de saisir comment ces hommes pensent et analysent leur rôle de père. Cette analyse repose sur des entretiens répétés menés avec 70 détenus, qui nous ont permis de proposer une typologie des paternités en prison se déclinant en quatre types : «marginale », « suspendue », «brisée » et « ressource».

$\underline{\text { Haut de page }}$

\section{Entrées d'index}

\section{Mots-clés :}

paternités plurielles, prison, devenir père, histoire familiale

\section{Keywords :}

$\underline{\text { multiple forms of paternity, prison, fatherhood, family history }}$

Haut de page 


\section{Plan}

$\underline{1 . \text { Introduction }}$

1.1. La méthodologie de l'enquête

1.2. Construire une typologie tridimensionnelle

1.3. Les résultats : quatre types de paternité des pères détenus

2. La paternité marginale

2.1. Une absence de préparation au devenir père, «j'étais un enfant avec un enfant »

2.2. La prison, une continuité, le «mode prison»

2.3. Une paternité vécue à distance, «je suis un père en carton»

3. La paternité suspendue

$\underline{3.1 . ~ D e v e n i r ~ p e ̀ r e ~ e n ~ p o i n t i l l e ́ ~}$

3.2. L'incarcération, une attente passive : «j'évite de trop y penser»

3.3. Des liens en suspens : «j'attends de sortir pour voir»

4. La paternité brisée

4.1. Se projeter dans la paternité : «il était voulu, il était désiré, très désiré »

4.2. Le choc de l'incarcération : " on est là comme un con»

4.3. Un sentiment d'illégitimité du rôle paternel : «c'est douloureux, je suis passé à côté »

$\underline{5 .}$ La paternité ressource

5.1. Devenir père et le rester

5.2. Expérimenter sa paternité en détention : «c'est un peu ma colonne vertébrale »

5.3. Un futur après la détention : «depuis que j'ai mon fils, je pense à la sortie »

6. Conclusion

Haut de page

\section{Notes de l'auteur}

Je souhaite remercier les membres de la revue ainsi que les évaluateur·rice's anonymes, pour le partage de leurs remarques et suggestions constructives dans l'élaboration de cet article. Une version préliminaire a également bénéficié des commentaires judicieux de Pascale Dietrich-Ragon et des doctorant·e's de l'Ined.

\section{Texte intégral}

$\underline{\text { PDF }} \underline{\text { Signaler ce document }}$

\section{Introduction}

Je présente ma grille d'entretien à la psychiatre qui est responsable de mon stage au Service médico-psychologique régional. Elle la lit soigneusement, sa lecture est entrecoupée de pouffements et de sourires. Elle pose les feuilles et me regarde : «M., tu sais, les détenus ils n'en ont rien à faire de leurs enfants, c'est purement utilitaire, ils vont te dire qu'ils sont de pauvres détenus qui ont besoin de leurs enfants, mais ils ne peuvent pas penser à autre chose qu'à eux, sinon tu penses qu'ils seraient pas ici. »

(Extrait de mon journal de terrain, 2012) 
[Conseiller pénitentiaire d'insertion et probation] — Il veut une permission pour voir son enfant.

[Procureure de la République] — C'est quoi ces gars qui veulent s'occuper de leurs gamins maintenant. J'aime ceux qui veulent être des bons pères, d'un coup ils se réveillent en prison et les gosses comptent » [ironie].

[Juge d'application des peines] — Dès qu'ils sont en prison, la fibre paternelle se réveille, c'est assez extraordinaire. J'ai plus de facilité à le croire pour Dieu, le truc de rencontrer Dieu...

(Extrait d'une commission d'application de peines, 2016)

- 1 La circulaire du 20 février 2012 relative au maintien des liens extérieurs des personnes détenues $\mathrm{p}(\underline{\underline{(. . .)}}$

1Les politiques pénales 1 affichent leur intention de maintenir les liens familiaux, considérés comme une condition essentielle de la réinsertion des détenus et de la prévention de la récidive. Mais sur le terrain, j'ai été confrontée à des réactions variées et souvent passionnées, voire à l'absence même de questionnement chez certain'e-s professionnel·le·s. J'ai parfois pensé qu'à certains égards, c'était la présence de la chercheure qui faisait émerger et exister ce sujet dans les discours. Si la question paternelle n'est pas invisible, elle oscille pour l'institution entre deux pôles : le soupçon prégnant de la mauvaise paternité et de son instrumentalisation d'une part, le pari d'une ressource pour la réinsertion future d'autre part.

- 2 En Amérique du Nord, ces questions ont été abordées dès les années 1960 en termes de crise familial (...)

2Les extraits cités précédemment sont particulièrement critiques et mettent en lumière la défiance ironique de certains·e·s professionnel·le·s. Bien qu'extrêmes, ces discours rencontrés sur le terrain amènent à s'interroger sur la paternité du point de vue des détenus. Comment ces hommes face à une institution qui encadre et réglementent les liens à travers des dispositifs spécifiques (parloirs, unité de visite familiale, téléphone, courriers) vivent-ils leur paternité et la définissent-ils en prison? Bien que la population carcérale soit composée à $97 \%$ d'hommes dont plus de la moitié sont des pères, les études sociologiques sur la paternité en détention en France $\underline{2}$ sont rares(Cardi, Abdallah, 2014). À l'inverse, les recherches sur les femmes incarcérées privilégient le thème de la maternité car, comme l'indique Coline Cardi (2008), « derrière la catégorie impersonnelle de "détenue", se cache une femme, mais surtout une mère ». Dans ses recherches, la sociologue montre comment la maternité est pensée à la fois comme un levier de réinsertion et un bénéfice secondaire de protection (Cardi, 2014). L'institution carcérale (re)affirme la différence des sexes ; d'un côté, la maternité est survisibilisée (notamment avec la mise à l'écart des détenues-mères), de l'autre, la paternité est amoindrie au profit du statut de détenu qui cache celui du «mauvais mari » et du «mauvais père » (Ricordeau, 2008).

3Les recherches sur le lien familial en prison ont avant tout porté sur l'organisation familiale, les effets de l'incarcération sur la famille et les proches (Bouchard, 2007 ; Ricordeau, 2008 ; Touraut, 2012) et sur les détenus (Douris, Roman, 2014 ; Ricordeau, 2008). L'étude de Gwénola Ricordeau a conduit à réactualiser les questions sur les liens familiaux en prison. Elle donne à voir les sentiments et les solidarités qui s'expriment entre les murs. Elle décrit 
les difficultés de maintenir les liens, les visites et montre que les pères perdent davantage le contact avec les enfants que les mères. Marie Douris et Pascal Roman, quant à eux, ont mené une enquête quantitative, avec un volet qualitatif, qui permet d'appréhender la question d'être parent en prison. Ils décrivent à la fois l'exercice, l'expérience subjective et la pratique de la parentalité. La force de leur étude tient à son interdisciplinarité. Les auteur·e·s « proposent d'éclairer le même objet, celui des relations parents-enfants en détention, du point de vue des parents » (Douris, Roman, 2014, 37) à partir du champ du droit et de la psychologique clinique. Mes travaux s'insèrent à la suite de ces recherches et plus largement au sein de la sociologie de la famille.

4Ces dernières décennies ont été marquées par une évolution du sens et des attentes vis-à-vis des rôles et des statuts paternels et maternel. Il ne s'agit plus de parler de « la » paternité, mais plutôt des multiples façons d'être père - lesquelles dépendent de facteurs socioculturels, familiaux, conjugaux et personnels (Quéniart, 2008 ; Turcotte et al., 2001). Dans les discours - moins dans la pratique - ces pères valorisent le modèle du partage des rôles entre le père « gagne-pain » et la mère « au foyer » et l'idéal d'une coresponsabilité sur toutes les tâches, sans distinction avec les mères. Cet idéal de paternité se veut universel mais se retrouve davantage chez les pères des classes moyennes ou supérieures. Dans les milieux populaires, les pères semblent moins répondre aux normes contemporaines de la «bonne » parentalité (Martial, 2012). Or, à la différence des «nouveaux pères », ces pères populaires plus traditionalistes ne font pas l'objet de beaucoup d'études en sciences sociales. Si la paternité fait l'objet de changements, ces derniers se présentent différemment selon les classes sociales (Le Pape, 2005).

5La sociologie de la famille a montré l'intérêt de replacer les relations familiales au sein des trajectoires biographiques. Celles-ci ne sont pas fixées et «l'individu façonne tout au long de sa vie son entourage de parents et de proches en jouant sur les espaces, les distances et les proximités » (Bonvalet, Lelièvre, 2012, 11). Les analyses de Catherine Bonvalet et Éva Lelièvre $(2012,12)$ notamment s'appuient sur une approche à la fois relationnelle et biographique. La première repose sur l'idée que l'individu n'est pas isolé, mais pris dans un ensemble de relations familiales, amicales et professionnelles, la seconde met «en évidence l'importance des trajectoires passées sur les comportements individuels observés à un moment donné ». Ma recherche s'inscrit dans la continuité de ces travaux en abordant deux dimensions spatiotemporelles en entretien : l'histoire passée à l'extérieur et le présent de l'incarcération.

- 3 Comme le soulignent Marie Douris et Pascal Roman $(2014,84)$, le livret reçu en début d'incarcératio (...)

6Certaines enquêtes montrent la difficulté d'accéder à la parole des hommes sur la famille et notamment dans les milieux populaires (Le Pape, 2005 ; Martial, 2012 ; Schultheis et al., 2009). Les femmes restent encore les détentrices de l'histoire familiale : en attestent les études plus nombreuses sur les mères et la famille en prison (Cardi, 2008 ; Touraut, 2012 ; Joël, 2017). Étudier la paternité en prison permet l'accès à des situations limites, mais révélatrices d'expériences paternelles. L'étude montre également que l'institution carcérale tend à homogénéiser les détenus sans prendre en compte leur spécificité en tant que pères $\underline{3}$. Au-delà du vide sociologique à combler, s'intéresser à des paternités contraintes éclaire sur les différents aspects qui composent l'exercice de la paternité, son appropriation et la place qui lui est faite dans les trajectoires biographiques d'hommes. À partir d'une analyse des vécus pré-carcéral et carcéral, il s'agit de comprendre comment les contraintes (matérielles, sociales 
et familiales) conduisent les pères à estimer ce qu'ils peuvent et ne peuvent pas faire en tant que parent et comment cela influence la perception de leur paternité. Bessin et al. $(2009,172)$ expliquent qu'un même événement — devenir père/être incarcéré — qui se produit à des moments différents dans une vie ou qui concerne des personnes socialement distinctes n'aura certainement pas les mêmes conséquences. L'objectif de cet article est donc d'analyser la diversité des relations parentales et les différentes postures paternelles que les détenus organisent, préparent, questionnent et subissent. Pour présenter les éléments principaux qui composent les paternités en prison, je m'appuierai sur une typologie construite à partir d'entretiens centrés sur les interactions au parloir.

\subsection{La méthodologie de l'enquête}

7Cet article se fonde sur une enquête réalisée entre 2012 et 2014 et un travail de doctorat en cours sur la paternité et les relations aux proches des hommes incarcérés. Je m'appuie ici sur des entretiens réalisés avec 70 détenus dans deux maisons d'arrêt (MA) et deux centres de détention $(\mathrm{CD})$.

- 4 Si un individu commet un inceste, une violence envers l'enfant ou tue l'autre parent, il est déchu $(\ldots)$

- 5 Dans mon courrier, j'expliquais mon souhait d'échanger avec eux sur leur relation à leur(s) enfant ( (...)

8Le recrutement de l'échantillon de l'étude s'est opéré grâce aux soignant·e·s du Service médicopsychologique régional (SMPR) et les conseiller-e·s d'insertion et de probation (CIP). J'ai énoncé quelques critères préalables pour sélectionner les détenus : être père et ne pas avoir commis d'acte incestueux, de violence envers ses enfants ou ne pas être incarcéré pour meurtre envers l'autre parent $\underline{4}$. En centre de détention, les détenus ont reçu une lettre décrivant l'enquête. Ils pouvaient refuser d'y participer en renvoyant un courrier au CIP $\underline{5}$. En maison d'arrêt, je les ai rencontrés individuellement pour leur présenter mon étude. La motivation des détenus à participer à mon enquête se décline selon quatre aspects : lutter contre l'ennui carcéral, profiter d'un moyen de se confier, instrumentaliser l'entretien (se faire bien voir, espérer bénéficier d'avantages), obtenir une reconnaissance et se valoriser (en se différenciant de la masse carcérale).

- 6 Avant chaque nouvel entretien, je lisais le(s) précédent(s) entretien(s) pour avoir en mémoire les (...)

- 7 En master, j'ai utilisé le « vous » lors du premier entretien mais j'ai proposé rapidement aux enqu $(\ldots)$

9Les enquêtés ont été vus en moyenne trois fois, me permettant d'aborder plusieurs aspects de leur paternité. Répéter les entretiens est une méthodologie lourde et contraignante $\underline{6}$, mais qui m'a semblé nécessaire pour créer un lien de confiance. La relation sociologique est une relation particulière qui est amenée à se construire sur un temps long pour en obtenir tous les bénéfices. J'ai exclu l'entretien unique, une condition peu propice au récit de soi en détention, car la méfiance fait partie intégrante de la recherche. Rencontrer plusieurs fois les enquêtés favorise leur sensibilisation à la technique d'entretien et permet de dépasser le discours conventionnel. Le premier entretien a débuté par une question ouverte « qu'est-ce qui compte pour toi 7 aujourd'hui, qu'est-ce qui est le plus important ou te motive au quotidien ? ». Cette question «brise-glace » (Kaufmann, 2011) m'a permis dans un premier temps d'accéder à un récit moins engageant, en me plaçant dans l'actualité biographique de la personne. En 
obtenant la participation de l'enquêté, j'ai pu introduire progressivement mon objet d'enquête. Dans un premier temps, j'ai appréhendé l'entrée dans la paternité et la nature du lien conjugal. Par ce biais, j'ai identifié les unions multiples possibles, replaçant chacun des enfants dans des histoires familiales singulières. Par la suite, j'ai établi des liens entre le pré-carcéral et l'incarcération afin d'entrevoir les tensions et les logiques d'adaptation entre le vécu passé et la situation présente.

- 8 Je me suis rendue plusieurs fois au parloir pour réaliser des entretiens avec les surveillants. J'a (...)

10Mon analyse se fonde sur des discours de pratiques au parloir et non pas sur l'observation participante $\underline{8}$. Ce choix s'explique par la dimension concrète et saisissable de ce lieu. Les pratiques contraintes pourraient sembler homogènes. En interrogeant les détenus sur les visites, j'ai tenté d'en appréhender plusieurs aspects : quelle place prennent-elles dans l'expérience parentale ? Comment s'inscrivent-elles dans leur vie quotidienne en prison ? Et comment sont-elles racontées à la chercheure ? Cette dernière question n'est pas anodine dans la production des données. L'enquêtrice, une femme blanche, de 22 ans, a pu renvoyer l'image d'une « bonne parentalité » attendue (être un père présent au quotidien, hors de prison, pourvoyeur, chef de famille, etc.). Aucun des détenus dont les liens se sont distendus depuis des années n'a exprimé un renoncement franc. Ils conditionnent essentiellement la reprise de la relation à la sortie de prison, mais il n'est pas certain que cet événement marque la reprise de ce lien. Cependant, il semble difficile d'exprimer le refus (voire le désintérêt) de (re)voir ses enfants à une chercheure qui s'intéresse aux liens père-enfant. L'étude de la paternité en prison constitue donc l'assemblage de multiples fils reliés entre eux (Elias, 1997, 223) : la mise en récit de soi, le type de père que ces hommes étaient avant et sont pendant l'incarcération et enfin leur ressenti et la signification du rôle paternel pour eux.

\subsection{Construire une typologie tridimensionnelle}

Les éléments principaux pris en compte pour construire cette typologie

1) L'entrée dans la parentalité : le récit de la première naissance puis des suivantes ; le désir d'enfant. La situation conjugale/relation à la mère au moment de la naissance ; la relation à l'enfant avant la prison et les pratiques parentales.

2) Le moment de la prison : la relation à l'enfant et à la famille en prison ; les changements perçus dans la relation; le vécu de la peine.

3) Les registres discursifs utilisés pour se raconter (héroïsation, occultation et évitement, tragique, analytique et politique). Le rapport au temps passé, présent, futur (de l'ancrage dans le moment présent à la capacité de se projeter dans le futur); la perception de sa paternité et la manière de se présenter en tant que père.

De ces éléments ont émergé des profils me permettant de dégager certaines spécificités : profil pénal (infraction et récidive), type d'établissement, âge, etc.

11Les typologies de Gilles Chantraine (2004) et Coline Cardi (2008) dont j'ai pu m'inspirer sont construites à partir du rapport à l'institution, de la trajectoire pénale, de la dialectique délinquance/répression et du milieu et de l'origine sociale. Mon intérêt se porte 
principalement sur la trajectoire parentale, utilisant la trajectoire pénale comme une variable secondaire. Pour expliquer la pluralité des paternités en prison, j'ai construit une typologie pluridimensionnelle issue de mon travail de master et de thèse ainsi que des entretiens semidirectifs. L'ambition est d'intégrer trois dimensions complémentaires : tout d'abord une approche biographique qui permet d'accéder à un discours diachronique de l'histoire familiale et conjugale avant l'incarcération des enquêtés ; ensuite une analyse relationnelle de la paternité en prison telle que les personnes interrogées décrivent leurs pratiques parentales concrètes à travers les différents dispositifs (parloir, unité de visite familiale [UVF], téléphone, permission, etc.) et tel que le lien père-enfant s'inscrit au sein d'un ensemble de relations incluant le rôle de la mère, de la famille d'origine, des co-détenus; enfin dans le cadre d'une analyse compréhensive (au sens wébérien du terme) la façon dont les pères décrivent leurs rôles, «pensent » les normes de paternité, se situent personnellement sur ce qui fait un père et finalement comment ils se jugent eux-mêmes.

\subsection{Les résultats : quatre types de paternité des pères détenus}

- 9 Récapitulatif des caractéristiques de chacun des types en Annexe ainsi que des boxplots qui permett (...)

12Les conditions d'incarcérations tendent à uniformiser et confondre les détenus. Pourtant, ce qui frappe, c'est la diversité des situations. La chercheure se trouve face à un foisonnement. Les hommes rencontrés sont tous pères, mais décrivent différentes entrées dans la parentalité allant de la paternité accidentelle à la paternité anticipée ; certains sont devenus pères avant la prison et d'autres pendant ; certains ont des visites et d'autres n'en bénéficient pas ; les pères peuvent avoir plusieurs enfants de mères différentes ou d'une mère unique. $D$ 'autres encore décrivent l'effet perturbateur des visites dans leur peine carcérale, tandis que certains en font au contraire une ressource essentielle. Les exemples peuvent être multipliés. Cette typologie révèle l'existence de certaines constances et régularités. Les situations prennent sens dans une contextualisation et une analyse spatiale (sphère publique/privée) et temporelle (passé, présent, futur). Cette typologie se décline selon quatre types de paternité que je propose de nommer : marginale, suspendue, brisée et ressource 9 . Pour les illustrer, je présenterai un ou deux exemples significatifs qui reprennent l'essentiel des caractéristiques de chaque type.

\section{La paternité marginale}

- 10 Comme le décrivent Marie Douris et Pascal Roman $(2014,108)$, j’ai pu faire le constat que l'exercic (...)

$13 \mathrm{Au}$ cours de cette recherche, les enquêtés ont souvent marqué l'opposition entre le père du « dedans » et celui du « dehors » pour montrer leur impossibilité de se sentir père comme avant. L'incarcération aurait pour effet de modifier les rôles parentaux. Gwénola Ricordeau (2008) a interrogé l'impact de la prison sur les liens affectifs, mais donne peu d'éléments sur la vie passée des détenu·e·s. Dans mon étude, je constate cependant dans certains cas un très faible écart entre le pré-carcéral et le carcéral : c'est ce que je nomme la paternité marginale. Le choix du terme de paternité marginale renvoie à deux aspects : à la fois un mode de vie atypique tourné vers la délinquance (business dans les stupéfiants, vols et braquages surreprésentés) et une paternité vécue à distance et peu expérimentée dans des pratiques 
quotidiennes (loisirs et prise en charge des différents besoins de l'enfant). Les détenus, sans être ni dépossédés de leur paternité ni complètement exclus, jouent peu leur rôle paternel et l'exercice de ce dernier dépend essentiellement d'un tiers (compagne et famille) $\underline{10}$.

\subsection{Une absence de préparation au devenir père, «j'étais un enfant avec un enfant»}

14Les détenus qui peuvent être rapportés à l'idéal type de la paternité marginale se caractérisent par un projet parental peu anticipé, qui, selon eux, leur «tombe dessus ». Devenus pères avant 25 ans, leur vie est surtout orientée vers l'amusement, le business et la vie « au jour le jour ». L'enfant serait issu davantage d'un désir maternel ou d'un manque de vigilance contraceptive que les enquêtés attribuent aux mères. Certains investissent toutefois le rôle traditionnel de père pourvoyeur, justifiant ainsi leur activité délictueuse. La paternité marginale regroupe pratiquement la moitié des détenus rencontrés (31 sur 70). Cette proportion s'explique par la surreprésentation d'enquêtés jeunes en maison d'arrêt (41 détenus interrogés sont en maison d'arrêt dont 22 classés dans ce type de paternité) pour les délits suivants : trafic de stupéfiants, vol, escroquerie, violence et violence conjugale. Ces hommes se rapprochent de la « clientèle pénale » (Cardi, 2008, 253) où l'incarcération apparaît comme une fatalité, « l'aboutissement d'une trajectoire de galère » (Chantraine, 2004, 14) rythmée par la « débrouille», les «trafics » et les petits boulots ponctuels (Cardi, 2008, 254).

- 11 L'UVF est un appartement meublé de deux ou trois pièces, séparé de la détention, où la personne dét (...)

15Pour illustrer la première dimension qui a permis de construire cette typologie, la biographie individuelle, je présenterai deux enquêtés : Jawad, incarcéré en centre de détention, et Bilal, en maison d'arrêt, tous deux reconnus coupables de trafic de stupéfiants. Jawad a 30 ans, il est marié et vit en couple depuis 10 ans avec Linda. Il a deux enfants, Amine (5 ans) et Malik ( 3 ans), ce dernier conçu lors d'une visite en unité de visite familiale (UVF)1․ Il explique qu'il ne voulait pas avoir de deuxième enfant « mais elle est tombée enceinte, je crois qu'elle le voulait. [...] De toute façon fallait bien qu'elle s'occupe pour qu'elle m'attende, je ne sais pas... avec une longue peine comme ça ». Incarcéré depuis 4 ans, il a été condamné à une peine de 8 ans et 8 mois. Très investi dans son business de stupéfiants, il s'est peu occupé de son premier fils.

— Comment tu as vécu l'arrivée du premier enfant?

— Je l'ai vécu normal, je ne sais pas, elle était enceinte, elle a accouché, ça a rien changé pour moi j'étais toujours dans mes trucs, dans ma vie illicite que je faisais. [...]

— Quel type de père tu étais avant la prison?

_ J'étais pas un père, je savais que j'avais un enfant... la prison m'a fait changer.

— Tu t'en occupais ?

— Non je vais pas mentir, je faisais rien du tout, j'étais presque jamais là et quand j'étais là c'était que le soir et je regardais la télé. 
(Jawad, 30 ans, concubinage, 2 enfants [ 3 ans et 5 ans], incarcéré depuis 3 ans)

- 12 Selon l'étude sur la Précocité et instabilité familiales des hommes détenus (Cassan, Mary-Portas, 2 (...)

16Pour Jawad, devenir père n'a pas marqué un changement décisif, il a vécu cette expérience à distance et n'a pris conscience de sa paternité qu'en prison. Pour lui, faire un enfant s'inscrit dans la «normalité », une suite logique dans la relation, mais qui ne modifie pas fondamentalement son mode de vie. Jawad, devenu père jeune, inclut difficilement sa paternité dans sa biographie personnelle. Olivier Galland (2011) identifie trois moments qui caractérisent la transition à l'âge adulte : le début de la vie professionnelle, le départ de la famille d'origine et le mariage. La naissance d'un enfant serait le dernier palier de cette transition (Goyette et al., 2012). Cependant, d'autres études ont montré que les dynamiques de constitution de la famille ont changé et deviennent complexes. Les parcours de vie individuels sont de moins en moins uniformes et se diversifient de plus en plus (Pailhé et al., 2013, 2). Les hommes incarcérés ne sont pas à l'abri de ces évolutions biographiques et les vivent d'autant plus fortement qu'ils sont exposés à la précarité et aux discriminations. Les paternités marginales concernent des hommes jeunes qui vivent généralement des expériences plus tôt que la population générale. Cette accélération biographique conduit à précipiter ou réaliser ces étapes précocement, rendant difficile l'appropriation de certains projets et notamment le projet parental $\underline{12}$.

17Bilal décrit au cours des entretiens la précocité de sa paternité. Il a eu deux enfants d'une première union : Sami (l'aîné) et Salim (12 ans). Sami est décédé d'un accident en vacances alors que Bilal est incarcéré. À la suite de cet événement, il divorce et se remet en couple avec sa compagne actuelle avec laquelle il a deux enfants, Ali ( 2 ans et demi) et Larbi (4 mois), tous deux conçus au parloir. Salim et Larbi n'étaient pas voulus. Bilal était incarcéré à la naissance de Sami, Salim et Larbi, puis en cavale lors de celle d'Ali. Il ne se sentait pas prêt à devenir père : " ça faisait bizarre, on dirait que j'étais un enfant avec un enfant, j'ai eu 20 ans, 12 jours après sa naissance. [...] Entre 20-25 ans, c'était la jeunesse, on sort en boîte et puis je buvais pas mal ». Depuis 2008, il enchaîne les incarcérations : «je n'ai pas été beaucoup dehors depuis ». Il a vécu très peu avec ses enfants, «j'ai pas beaucoup de souvenirs avec Ali et Larbi. Ali, il a marché le jour où je me suis fait interpeller et entre-temps je ne suis pas sorti. Je suis en train de faire une peine de 4 ans donc normalement je sors en 2017, mais j'espère avoir un aménagement. En tout j'ai pris 7 ans et j'ai fait 34 mois ». Entre le $1^{\text {er }}$ et le $4^{\mathrm{e}}$ entretien, une autre peine s'est ajoutée, laissant présager une sortie fin 2018 . Au cours du $3^{\mathrm{e}}$ entretien, je reviens sur son projet de paternité.

Mon premier fils c'était un projet, Larbi c'était un accident. C'était surtout ma femme qui voulait, elle avait... c'était en 2010, elle avait 22 ans, elle me prenait la tête, elle me disait «j'ai pas de vie, tu es en prison » donc on a fait un bébé parloir. Salim ce n'était pas voulu, je fais que des garçons. Elle voulait un enfant, elle ne voulait pas attendre.

- 13 Âge du dernier et du premier enfant.

(Bilal, 33 ans, concubinage, 4 enfants [4 mois à 12 ans $\underline{13}$ ], incarcéré depuis 34 mois)

18Bilal est déjà père, mais sa nouvelle compagne n'a pas d'enfant. L'extrait permet d'entrevoir une différence dans le désir d'enfant : pour lui, il a une valeur utilitaire qui permet de maintenir le couple et de répondre à la demande de sa conjointe de donner du sens à cette 
existence. Il voit la motivation de sa femme comme une affirmation de soi et le prolongement de leur histoire commune. On peut supposer qu'être mariée ou mère lui apporte une identité, une légitimité (Schwartz, 2012, 220), et inscrit le couple dans un futur possible. Bilal incarcéré, les perspectives d'avenir sont faibles. Il se positionne en arrière-plan, acteur secondaire de ce projet d'enfant.

19Ces deux exemples montrent le peu d'anticipation dans le projet de devenir père. Cet aspect s'inscrit plus généralement dans une perception de l'avenir à court terme. Les enquêtés oscillent entre deux états - ce que Hamel $(2003,162)$ nomme « un statut "ni enfant, ni adulte" » Sans pour autant se désintéresser de leur statut parental, ils le vivent parfois de manière périphérique notamment au jeune âge de l'enfant, se déchargeant un temps sur la mère et la famille.

\subsection{La prison, une continuité, le « mode prison»}

- 14 Se référer pour chacun des types aux bloxplots qui permettent de voir les dispersions.

- 15 Seuls deux détenus sur les 10 n'étant plus en couple voient de manière irrégulière leurs enfants gr $\underline{(\ldots)}$

20Certains pères rationalisent la pauvreté des liens familiaux du fait de la détention. D'autres continuent à être le pourvoyeur quitte à faire du trafic en détention, ne remettant que peu en question leurs activités délinquantes. La paternité marginale regroupe de jeunes pères avec des enfants également jeunes (la médiane est de 10 ans pour le premier et 3 ans pour le dernier)14, parfois issus de plusieurs unions. L'incarcération éloigne la paternité, surtout pour les hommes séparés et divorcés, et ne semble apparaître qu'à de rares moments, tel le parloir pour ceux encore en couple15. La paternité, loin d'être une ressource, affaiblit les stratégies d'adaptation à la prison.

21En interrogeant Jawad sur la visite au parloir, il marque la dissociation entre ce moment précis et le temps de la détention.

— Vous faites quoi pendant le parloir?

— On est là, on parle, on rigole, on joue. Ils me racontent leurs trucs. Dans un parloir on ne peut rien faire.

— Ça te fait plutôt du bien ou du mal de les voir?

— C'est les deux, au début c'est bien après les petits, puis ils pleurent ils ne veulent pas partir.

— Tu te sens comment?

- Après dès que je rentre ici je me remets en mode prison, faut pas trop penser, rester sur un nuage c'est pas bon, faut revenir à la réalité, c'est comme ça direct.

— Tu cherches à ne pas y penser?

- Pour avancer, pour pas être déçu, pour pas... je sais pas comment expliquer. C'est pour pas être déprimé que je me mets en mode prison sinon tu commences à réfléchir, c'est pas bon. 
— Tu cherches à oublier que tu es père ?

- Oui, il faut que je me dise que je suis un détenu ou un prisonnier, je me dis pas que je suis père, ici on est juste un numéro, on est pareil.

- 16 Âge du dernier et du premier enfant et temps d'incarcération lors du dernier entretien.

(Jawad, 30 ans, concubinage, 2 enfants [3 ans et 5 ans], incarcéré depuis 3 ans $\underline{16}$ )

22Le parloir ne permet que très partiellement (voire pas du tout) de pallier l'absence de Jawad et d'assoir son rôle de père. Le lieu contraint la rencontre et empêche de déployer la variété des fonctions parentales (Carlson, Cervera, 1991). Les sentiments vécus sont ambivalents, restreints et se cantonnent à ce lieu. Le «mode prison » conduit à jouer un rôle attendu en détention et y rend difficile la superposition des territoires (notamment du parloir à la détention et inversement). Dans le quotidien carcéral, il s'agit de faire abstraction de sa paternité pour supporter au mieux la peine d'emprisonnement. En précisant qu'il « faut revenir à la réalité », Jawad sous-entend que la réalité n'est pas le parloir, mais ce qui se joue en détention. Pour les détenus classés dans la paternité marginale, l'incarcération rend complexe l'implication dans sa paternité. L'événement ponctuel du parloir représente « autant d'occasions de leurrer sa propre perception du temps carcéral » (Chantraine, 2004, 168) et s'inscrit difficilement dans le quotidien de l'individu. Oublier le dehors paraît nécessaire pour survivre dedans. Cet événement souvent décrit comme une «bouffée d'oxygène » conduit aussi à exposer ses faiblesses. Gwénola Ricordeau $(2008,81)$ explique que le parloir trouble et « déborde sur la vie quotidienne ». Ce lieu pourrait représenter en prison un espace privé où l'intimité et l'affect peuvent émerger tandis que ces comportements sont réprouvés hors de ce lieu. Cette situation conduit à un morcellement identitaire et à une perpétuelle mise en tension de soi d'un lieu à l'autre.

- 17 Dans l'ouvrage collectif sur les sorties de délinquances (Mohammed et al., 2012). Vaughan montre qu (...)

23Bilal distingue également les lieux en détention. Il explique qu'il essaye de ne pas penser à dehors : «je ne pense qu'à la prison, j'attends que ça passe ». Il ajoute qu'en « prison faut oublier l'extérieur, [...] ça ne sert à rien » d'y penser. Il déclare parler peu de sa famille en détention et se focalise sur d'autres sujets : «nous, en promenade, on parle d'abord de l'intérieur puis après de l'extérieur, "tu as mangé quoi : une pizza, un gâteau ?" C'est un moyen de survivre, de ne pas se faire du mal. La dernière fois en promenade, on était trois trafiquants de stups qui font des go fast en Espagne et on se disait par où on passe, comment on travaille, chacun a sa manière de faire [...] Les hommes en général, ils parlent pas trop de leur famille. J'ai des enfants, basta, surtout entre hommes on parle de tout et de rien, mais pas de nos familles ». Il cantonne sa famille au parloir et partage peu ses souvenirs familiaux avec d'autres détenus. Pour autant, il mêle sa famille à ses pratiques délictueuses. Pendant un entretien, il explique que les enfants sont un motif d'arrêt de la délinquance $\underline{17}$, mais sa paternité, depuis longtemps, est imbriquée dans un parcours d'incarcération auquel il aimerait se soustraire. Son discours souvent ambivalent est en décalage avec la continuité de ses pratiques.

- 18 Bilal explique qu'il ne trafique jamais en présence de sa mère, sûrement pour ne pas la mêler à son $\underline{(. . .)}$ 
— Le rôle du père, c'est de nourrir ses enfants et la mère, c'est la patronne du foyer. [...] Pour moi c'est normal, chacun son boulot. [...] Je fais de l'argent en prison alors dehors ce sera facile. J'aime faire de l'argent, je sais que je suis là, je ne suis pas mort, ça m'occupe. Quand je vais en parloir et que j'ai rien, je suis dégoûté, j'ai l'impression de m'être fait arnaquer. Ça sert à rien, c'est comme si j'avais eu parloir pour rien $\underline{18}$. Je ne passe pas à la douane sans rien. Quand ma mère vient le samedi, j'ai rien, alors en salle d'attente je propose aux mecs de faire passer et on me paye cinquante euros. Je suis payé cinquante euros la minute.

— Ça ne dérange pas ta femme de passer?

— Bah... ça l'arrange, c'est elle qui récupère l'argent. Je me suis mis avec elle quand j'étais en prison en 2013, elle savait ma réputation, elle savait ce que je faisais [...]. Ça fait mes mandats et ça aide un peu ma femme, au moins je ne sers pas à rien. $\mathrm{Y}$ a des mecs qui ont rien et en plus ils demandent à leur femme.

(Bilal, 33 ans, concubinage, 4 enfants [4 mois à12 ans], incarcéré depuis 34 mois)

24Bilal décrit les rôles parentaux distincts, justifiant par la même occasion son business pour aider femme et enfants. Il est le pourvoyeur, tandis que la mère s'occupe du foyer en son absence. Cette différence des rôles s'opère d'autant plus dans les milieux les moins favorisés (Schwartz, 2012). Par son trafic, il subvient à leurs besoins et amoindrit son sentiment d'inutilité. Disposer d'argent protège de la honte d'être à la charge de sa famille.

25L'expression « en mode prison » se définit à la fois par un vécu de la détention concentré sur la camaraderie virile (blagues et humour tendancieux) que je n'ai pas développé ici, par un « hexis de délinquant » (Chantraine, 2004, 86) (valorisation du parcours délictueux, connaissance de la prison, pratique du sport) et par la prédominance du moment de l'incarcération dans le récit de soi. Dans ce contexte, être père a peu de prise sur ce vécu.

\subsection{Une paternité vécue à distance, « je suis un père en}

\section{carton $»$}

26Les deux aspects précédents de la paternité marginale permettent d'entrevoir la difficulté de se sentir père en détention quand l'implication parentale passée était faible. La réflexivité sur leur rôle montre une certaine lucidité des hommes rencontrés sur ce qu'ils ne font pas et n'ont jamais fait. L'exercice de la paternité n'a pas sa place en prison, il est renvoyé à l'aprèscarcéral.

27Jawad voit ses enfants toutes les trois semaines au parloir, en partie à cause de l'éloignement géographique de la prison, rendant d'autant plus difficile l'appropriation de son rôle :

Je ne suis pas un père, je ne suis jamais là, je ne peux pas faire mon rôle de père, je suis un père en carton, je ne suis pas un père. Je suis un père toutes les trois semaines quand ils viennent au parloir. [...] Je suis à moitié père parce que je ne remplis pas mon rôle de père, je ne fais rien du tout de père, père c'est beaucoup de responsabilités. Je sers à rien, c'est vrai, je ne sers à rien. Même si je sais que dehors, y en a qui servent à rien, faut que je sois dehors.

(Jawad, 30 ans, concubinage, 2 enfants [ 3 ans et 5 ans], incarcéré depuis 3 ans) 
28L'enquêté décrit un sentiment d'inutilité. En utilisant l'expression de «père en carton » il représente la fragilité de son rôle : il «ne sert plus à rien » et ne remplit plus ses fonctions. L'entretien met aussi en exergue la différence entre « être père » et «accomplir son rôle de père ». Dans la littérature anglo-saxonne, on distingue les usages des termes parenthood et parenting. Le premier terme désigne la condition de parent, un statut attribué par la reconnaissance juridique de l'enfant, et le second signifie être parent par ses pratiques, par la prise en charge et les responsabilités relatives à l'enfant. La prison agit avant tout sur le parenting, mais semble avoir un impact plus général sur l'appropriation du parenthood. Dans le cas de Jawad, le fait de ne pas remplir son rôle de père par sa présence et d'assumer les responsabilités qui incombent à son rôle (parenting) l'empêche de s'approprier son statut de père (parenthood).

29Pour les pères regroupés dans la paternité marginale, l'incarcération rend visible à la fois leur faible implication parentale avant et pendant l'incarcération et la prise en charge des rôles familiaux par les femmes. Les rôles anciennement partagés tendent à s'amoindrir.

— Quand Linda prend des décisions, elle t'en parle?

- Non, je crois qu'elle prend ses décisions sans moi, depuis le temps, je suis au courant de presque rien, ça y est c'est comme si je servais à rien, j'ai pas le choix, je vais pas la forcer, je ne sers à rien, je suis un boulet un peu.

- Avant, vous en parliez ensemble?

— Y a pas trop de communication bizarrement. Avant on parlait un peu plus, je ne sais pas... Avant je faisais ma vie, j'étais jeune. Pour moi j'étais prêt (à être père), mais en vérité j'étais pas prêt, normalement pour être prêt faut avoir stoppé les trucs illégaux, j'étais encore dans mon délire.

(Jawad, 30 ans, concubinage, 2 enfants [ 3 ans et 5 ans], incarcéré depuis 3 ans)

- 19 Marie Douris et Pascal Roman (2014) font le même constat dans leur étude : si «l'incarcération ne $(. .$.

30En comparant sa situation pré-carcérale et actuelle, Jawad perçoit l'absence de communication avec sa compagne, la prison a simplement contribué à renforcer cet état de fait. Son mode de vie entraine des difficultés certaines à s'engager dans son nouveau rôle et à se sentir responsable des autres. Son manque d'implication accentue ce sentiment d'inutilité. Il estime être une charge, en se décrivant comme un «boulet». Dans ce contexte, les décisions parentales sont assumées par la mère ou un tiers. Pour Jawad, l'incarcération révèle sa difficulté à investir son rôle paternel que le parloir ne permet pas de construire ou de reconstruire. Si ce lieu favorise l'expression de la paternité, il relève d'autant plus la fragilité des liens. Les détenus déjà peu impliqués avant l'incarcération maintiennent cette posture 19.

— Et vis-à-vis de tes enfants, tu es comment en prison avec eux ?

- On dirait en prison que mes enfants sont mes enfants, mais que je suis mort en prison, que je peux rien faire pour eux, que je peux que parler avec eux, mais que ça sert à rien un mort qui parle. Par exemple, même ma femme me demande de l'engueuler, moi je lui dis « je peux pas, je suis en prison, je le vois jamais, une fois par semaine et encore, je peux pas 
l'engueuler, moi », je lui dis, je peux pas, je suis en prison, je le vois jamais, une fois par semaine et encore je peux pas m'engueuler avec lui.

— Tu vois une différence avec ce qu'il y avait avant la prison et maintenant ?

- Y a aucune autorité en prison, on ne peut pas engueuler son fils alors qu'on le voit jamais, seulement trente minutes dans la semaine.

(Bilal, 33 ans, concubinage, 4 enfants [4 mois à 12 ans], incarcéré depuis 34 mois)

- 20 Dans l'étude de Marie Douris et Pascal Roman $(2014,66)$, on constate que « $69,7 \%$ enfants âgés de 1 (...)

31Bilal considère son incarcération comme la mort sociale de son rôle. Le lien avec ses enfants se réduit à son minimum, «je peux rien faire pour eux ». S'il reste le père de ses enfants, c'est le lien biologique qui semble maintenir son statut parental. Au cours de la visite, les détenus sont encouragés par leurs proches à investir certaines caractéristiques de leur rôle paternel, à commencer par l'autorité. Bilal est ainsi confronté à son illégitimité dans la position de celui qui gronde et ne peut accéder à la demande de sa femme. Dépendant des réactions de l'enfant 20 qui du jour au lendemain peut refuser les visites, il s'autocensure. N'ayant jamais investi ce rôle, il n'est pas davantage en capacité de l'assumer en prison et souhaite préserver une image favorable de lui-même. Il dresse donc le constat de ce qu'il ne fait pas, mais qu'il n'a finalement jamais fait.

32Les allers-retours en prison produisent une alternance entre présence et absence qui favorise le « décrochage » ou la « désertion » des pères (Martial, 2009 ; Régnier-Loilier, 2013). Turcotte et al. (2001) indiquent que le sentiment de compétence parentale et la valorisation de cet engagement s'expriment lorsque les pères prennent une part active aux soins, à l'éducation et ont des interactions directes avec leurs enfants. En l'absence de cette expérimentation du lien, ce dernier s'amoindrit face au vécu de la détention et de la délinquance. La prison révèle l'impuissance et l'étiolement des liens.

33Les pères que j'ai classés dans la paternité marginale peuvent paraître peu investis selon les normes dominantes du «nouveau père ». Leurs discours montrent une prise en charge des enfants limitée avant l'incarcération. Rappelons que « la rigoureuse division des rôles seule véritable constante dans les familles ouvrières - conduit les mères à assumer l'essentiel de la charge des enfants » (Schwartz, 2002, 145). Ce sont généralement les femmes qui représentent « le centre de gravité de cette sphère "privée" » (Schultheis et al., 2009). Leur histoire montre qu'en prison, certains hommes peuvent conserver un statut et une place dans la famille : le trafic, par exemple, est un moyen de soutenir les proches ; pour autant, le rôle de père est moins légitime. Les détenus ne décrivent pas ce rôle comme prioritaire ou constitutif de leur identité. De fait, l'emprisonnement affaiblit leur autorité pourtant primordiale dans leur discours. Ce sentiment dominant d'incompétence génère une certaine souffrance qui conduit les détenus à effacer leur paternité en détention. Pour supporter au mieux leur incarcération, ils mettent en place des stratégies d'adaptation au monde carcéral que j'ai nommé le « mode prison». Ils se concentrent avant tout sur le moment de l'incarcération et se projettent peu dans le futur qui « est trop lointain pour s'y attarder » (Schultheis et al., 2009, 160). La majorité des pères classés dans ce type sont relativement jeunes. Il n'est pas impossible que leur paternité prenne plus d'importance par la suite. 
D'autres reformeront de nouvelles familles qui s'inscriront différemment dans leur trajectoire personnelle.

\section{$\underline{\text { 3. La paternité suspendue }}$}

34La paternité suspendue se caractérise par une (quasi) absence de contact avec l'enfant pendant l'incarcération, rendant difficile l'inscription de la paternité dans une temporalité sociale vécue et éprouvée. L'incarcération est vécue comme un temps qui « manque d'alternative » (Schehr, 1999, 236). Le « présent suspendu » est un temps «que l'on subit plus que l'on gère ». Un parallèle est possible avec l'expérience du chômage décrite par Sébastien Schehr : il montre que cette expérience est proche de l'attente passive, " où il s'agit surtout d'attendre sans faire, d'exister sans vivre, de contempler un temps non rempli ». Ce parallèle fonctionne pour la paternité suspendue, que je décris comme un temps de latence.

\subsection{Devenir père en pointillé}

- 21 Arnaud Régnier-Loilier $(2013,73)$ indique que «plus la séparation est ancienne et plus la proporti (...)

35La paternité suspendue rassemble des situations plus complexes et diversifiées. Les détenus classés dans ce type sont au nombre de dix. Ce sont des détenus âgés d'une quarantaine d'années, présents majoritairement en centre de détention pour des peines relativement longues (huit détenus sur dix ont effectué une peine de plus d'un an et demi au moment de l'entretien). Les motifs d'incarcérations sont : mœurs, violence conjugale et dans une très moindre mesure infraction à la législation sur les stupéfiants et vol. Davantage que dans d'autres types, certains n'ont pas mentionné le motif d'incarcération. Le projet parental est tardif, comparé à la paternité marginale (après 30 ans), et les enfants sont plus âgés au moment de l'incarcération (10 ans pour le cadet et 11 ans pour l'aîné) que ceux des pères regroupés dans la paternité marginale. Le lien a pu se distendre davantage et depuis plus longtemps 21 .

36Le projet parental a pu être pensé et planifié, mais quelques naissances moins prévues sont survenues ; la majorité des détenus concernés par ce type ne sont plus en couple et l'ont été peu de temps. Ils pourraient être assimilés à des célibataires de longue durée. Certains ont vécu avec l'enfant avant l'incarcération, d'autres ont eu des contacts de manière discontinue. Pour illustrer la première dimension de ce type, je prendrai l'exemple d'André, 38 ans, père d'un fils de 11 ans. Avec son ex-conjointe, ils ont décidé d'avoir un enfant, avant de se séparer un an plus tard. Il dit ne pas savoir les raisons de cette rupture. "J'ai essayé de le voir, de le prendre le week-end, mais la mère ne voulait pas trop, je ne sais pas pourquoi ». Il est incarcéré en 2005 pour un délit dont il ne parle pas en entretien. Sa sortie est prévue pour 2023. Il attend 2009, année de son jugement, pour entamer des démarches, c'est-à-dire quatre ans après le début de sa peine. En 2010, grâce aux Relais enfant-parent, il voit son fils trois fois puis il est transféré. Il se met en relation avec SOS Papa pour avoir accès aux carnets scolaires de son fils, puis obtient du juge des droits de visite qui ne sont pas effectifs et le droit de l'appeler tous les dimanches au moment des entretiens (2014). André a très peu connu son fils avant l'incarcération. Au vu des obstacles, il se sent découragé. Il ne se sent pas capable de remotiver ce lien dans cet espace. Lahcen, quant à lui, a 47 ans et une fille de 11 ans : «elle est arrivée comme ça ». Son ex-conjointe a décidé de partir peu de temps après sa naissance et de lui laisser la garde, «c'était dur de l'avoir tout le temps ». Il a une peine de 
6 mois et récupérera la garde de sa fille à sa sortie. Pendant le temps de son incarcération, sa sœur s'occupe de sa fille. Malgré leur lien, il ne veut pas la faire venir en prison, mais maintient une relation téléphonique fréquente. Les points communs aux deux situations sont la suspension du lien physique avec l'enfant et une relation quasi inexistante avec la mère.

\subsection{L'incarcération, une attente passive : «j'évite de trop y penser»}

- 22 L'étude d'Aline Désesquelles et Annie Kensey $(2006,66)$ sur les liens familiaux confirme une corrél (...)

- 23 Gwénola Ricordeau $(2008,66)$ montre que certains détenus craignent d'être rejetés et, pour conserve (...)

37La paternité suspendue se vit en détention dans l'attente. Aucun des détenus concernés ne bénéficie de visite. En raison de facteurs personnels ou de contraintes liées à l'ex-conjointe, ces pères rencontrent des obstacles pour être en contact avec l'enfant. Deux situations se dessinent : dans l'une, ces pères n'ont pas d'interaction directe avec leurs enfants et ne les compensent pas grâce à d'autres moyens de communication comme le courrier ou le téléphone22. Les interactions sont quasi inexistantes. Dans l'autre, les enquêtés ont choisi de suspendre les interactions, mais continuent d'appeler la famille. Ils expliquent le souhait de ne pas confronter leurs enfants à la prison ou de leur éviter la contagion d'un stigmate carcéral qu'ils devraient assumer dans le quartier. Les détenus décrivent ainsi une suspension positive qui a pour fondement la protection $\underline{23}$. On peut aussi faire l'hypothèse qu'exclus par la famille, ils préfèrent affirmer être à l'origine de l'éloignement.

38Lahcen explique son choix de ne pas faire venir sa fille en prison :

Je ne veux pas lui dire pour la prison, j'attends qu'elle soit plus grande, je veux pas qu'elle soit perturbée, qu'elle ait de mauvaises fréquentations, qu'elle bascule et qu'elle s'habitue.

(Lahcen, 47 ans, séparé, 1 enfant [11 ans], incarcéré depuis 6 mois)

- 24 Par stigmatisation je me réfère à la définition d'Erving Goffman (1970) qui décrit ce processus com $\underline{(\ldots)}$

39En refusant le parloir, il tente d'éloigner sa fille de la détention. Faire venir son enfant en prison implique d'affronter le stigmate social24 dedans, mais également dehors (Touraut, 2012). Certains détenus décrivent leur peur de l'effet ricochet qui répercute l'image de détenu sur les proches. Ils craignent que l'expérience carcérale soit pour la famille plus douloureuse que de ne pas les voir.

40Face à ce type de paternité, les discours sur le lien père-enfant en prison sont peu étayés. Un extrait d'André fait sentir cette difficulté :

Il n'y a pas grand-chose à dire, je ne pense pas à grand-chose, j'évite de trop y penser en ce moment. Je préfère vivre au jour le jour plutôt que penser trop à ça, ça nous met mal à l'aise par rapport à la prison, si on pense de trop, on faiblit. On se renferme sur nous-mêmes, faut éviter de trop penser. 
(André, 38 ans, séparé, 1 enfant [11 ans], incarcéré depuis 9 ans, CD)

41L'entretien de Lahcen s'inscrit dans la même veine :

Je pense à plein d'autres choses, $\mathrm{y}$ a plein de gens ici qui font ça. $\mathrm{Y}$ a une routine, on est pris parce qu'il y a à faire même si y a pas grand-chose et on se focalise sur autre chose. Y a que le premier mois où c'est dur, où on dort pas et puis après on zappe. C'est un moyen de se protéger, autrement on vit pas, si on pense qu'à sa famille, ses enfants, je ne pense pas que j'y arriverais.

(Lahcen, 47 ans, séparé, 1 enfant [11 ans], incarcéré depuis 6 mois)

42Souvent exprimée de manière évasive, la paternité de ces hommes émerge en périphérie dans leur discours. Ils décrivent leurs stratégies pour meubler le temps carcéral et se détacher des problèmes liés à l'extérieur. Ce questionnement sur les liens à l'enfant semble apparaître avec la rencontre avec la chercheure. La situation d'entretien met au premier plan un sujet auquel ils ont peu réfléchi ou auquel ils ne veulent pas répondre. Les interactions sont ponctuées de phrases courtes et fuyantes. André, par exemple, élude les questions, justifiant qu'il ne sait pas y répondre ou qu'il n'y pense pas. Il n'a pas su m'éclairer sur son absence à l'accouchement (il ignore pourquoi la mère de l'enfant ne l'a pas prévenu), ni sur les motifs de la séparation du couple. Corinne Rostaing montre que certaines questions concernant la vie de couple et les enfants restent plus fréquemment sans réponse, car elles mobilisent un repère temporel (âge des enfants et années de naissance). Ce résultat peut s'expliquer par le fait que certains pères disposent de peu d'informations sur l'enfant depuis l'incarcération ou qu'ils ont peu ou jamais connu l'enfant (Insee, 2002, 95).

\subsection{Des liens en suspens : «j'attends de sortir pour voir»}

43La paternité suspendue se caractérise par un rôle paternel évoqué par touches, dans lequel les hommes se sentent peu engagés pendant l'incarcération. L'enfant est placé en périphérie des préoccupations, renvoyé à l'après-carcéral. Ils décrivent l'importance de se concentrer sur le vécu de la peine, mais dans un autre registre que celui de la paternité marginale. Les détenus se rapportant au premier type valorisent leur parcours délictueux, héroïsant leur capacité à contourner le contrôle de l'institution. L'incarcération ne questionne pas nécessairement leur place dans la famille : certains continuent à se considérer comme des chefs de famille, car ils restent des pourvoyeurs ou des figures d'autorité. Les détenus que j'ai regroupés dans la paternité suspendue ne sont pas dans le mode de l'intégration carcérale, ce que j'ai nommé le mode prison, mais tendent au contraire à faire « profil bas ». Ils ont des relations distendues avec une partie, voire l'ensemble du réseau familial, et évitent de nouer des relations au sein de la détention. Ils se situent davantage dans une logique de désaffiliation et cherchent à se défaire du stigmate lié à leur statut de détenu.

44La paternité est suspendue car ils sont dans une attente passive généralisée. Quand j'interroge André sur le sens de sa paternité, il se réfère au sens commun largement diffusé dans la société sur ce que doit faire un père (être responsable, consacrer du temps à l'enfant et assurer une présence quotidienne) plutôt qu'à sa propre expérience.

- Être père, c'est savoir s'occuper d'une personne, d'un enfant, avoir des responsabilités, être sérieux, montrer le bon exemple et savoir être là. 
— Et aujourd'hui, vous vous sentez comme tel?

— Bah pas trop, je le vois pas tellement, peut-être plus tard, petit à petit.

(André, 38 ans, séparé, 1 enfant [11 ans], incarcéré depuis 9 ans, CD)

45Les hommes concernés par ce type de paternité se sentent responsables de l'enfant, mais s'impliquent peu et surtout pendant l'incarcération. Leurs discours restent centrés sur leurs propres difficultés plutôt que sur les besoins de l'enfant. Selon eux, ils sont voués à attendre une meilleure situation.

- J'attends de sortir pour voir. Pour l'instant, j'ai pas tellement de projet, j'y pense pas encore. Dès que je sais la sortie, c'est là que je vais avoir mes projets et que je voudrais y penser.

— Est-ce que tu as parlé de la prison à ton fils ?

- Non, je ne sais pas, il m'a jamais posé la question, un jour je serai obligé de lui dire quand il sera grand. [...] Je ne suis pas avec lui, je ne sais pas exactement comment il se comporte, ce qu'il pense, j'attends de voir par la suite. J'attends, même si je voulais faire quelque chose ça servirait à rien, la juge fera rien, faut attendre.

(André, 38 ans, séparé, 1 enfant [11 ans], incarcéré depuis 9 ans, CD)

46La paternité suspendue se définit par la difficulté des hommes classés dans ce type à décrire leurs enfants, mais surtout leur rôle de père qu'ils n'expérimentent pas en prison. À la différence des hommes classés dans la paternité marginale, qui ont connu une accélération biographique et qui décrivent leur manque de préparation au statut de père, ceux classés dans la paternité suspendue vivent les événements plus tardivement ; ils sont plus âgés au moment de l'incarcération, mais également lors de la naissance du $1^{\mathrm{er}}$ enfant. Par ailleurs, la chercheure peine à recueillir dans les entretiens des éléments sur la manière dont ces hommes exerçaient leur paternité. Ceux qui justifient leur choix d'éloigner les enfants de la prison ressentent certainement moins de crainte quant à la reprise de leur rôle. Au contraire, ceux qui les ont peu connus avant et de manière discontinue ne luttent pas pour maintenir ces liens ou n'en ont tout simplement pas les moyens. Il leur faudrait une énergie qu'ils ne parviennent pas à mobiliser. Ce type se caractérise finalement par une certaine passivité imposée ou choisie. La relation co-parentale peu construite ou problématique joue pour beaucoup dans la définition de cette paternité. Le lien pourrait être brisé — s'il ne l'est pas déjà —, mais ils n'en font pas état, renvoyant leur paternité à un futur lointain.

\section{La paternité brisée}

47Le choix du terme de brisée témoigne de cette ambition parentale non réalisée à cause de l'incarcération et fait référence à une souffrance psychologique exprimée par les détenus. Leur modèle paternel se réfère à une quotidienneté et implique des responsabilités financières et affectives qu'ils ont l'impression de ne plus être en mesure d'assumer pleinement. 


\subsection{Se projeter dans la paternité : «il était voulu, il était désiré, très désiré »}

- 25 Ce type de paternité partage des points communs avec « la criminelle conforme » de Coline Cardi. L' (...)

48La paternité brisée regroupe quatorze détenus. Ils ont plus de 35 ans et avaient ou ont pour la plupart un emploi avant l'incarcération (10 sur les 14). Ils sont en couple ou ont vécu des relations conjugales longues (plus de 5 ans). Avoir un enfant était un projet anticipé ou, s'il n'était pas prévu, qui a pris un sens dans leur histoire personnelle. Cet aspect s'explique notamment par l'âge des détenus regroupés dans ce type. Ils ont pu construire sur le temps long un regard réflexif sur leur paternité, nourri par leur capacité de «mise en biographie » (Cardi, 2008, 301). Ils se sentent investis dans leur paternité à travers leur soutien financier, affectif et leur prise de décision dans la vie de l'enfant. Au contraire des détenus dont la paternité a été décrite comme marginale, les pratiques délictueuses ne s'inscrivent pas durablement dans leur trajectoire de vie. Ils vivent pour certains cette incarcération comme une erreur, ont été rattrapés par de vieux copains ou le passé d'une jeunesse perturbée (violence, absence des parents, délinquance environnante). Cette incarcération est vécue comme une catastrophe 25 qui impacte leur famille, leur travail, leur couple (séparation) et leur rôle paternel. Incarcérés majoritairement en maison d'arrêt, ils décrivent les conditions d'incarcération lamentables et «l'impossible familiarisation avec le temps, l'espace, les objets et les autres acteurs [nourrissant un] sentiment d'une irréductible étrangeté » (Joël, 2017).

49J'illustrerai mon propos avec l'histoire de Laurent, 38 ans. Il est incarcéré depuis 8 mois pour trafic de stupéfiants. Il a déjà effectué une peine pour le même délit, il y a 3 ans, d'une durée de 2 mois et demi. Marié depuis une vingtaine d'années, il a une fille de 17 ans et un fils de 14 ans. À la naissance de sa fille, il travaillait comme cadre ferroviaire. Il a ensuite opéré une reconversion pour devenir propriétaire d'un bar : «je travaillais non-stop, des journées pleines et après, par la suite, j'ai regretté. Les deux premières années, j'ai pas profité, j'ai eu les boules. Avec mon fils, c'était différent, il est arrivé 4 ans plus tard et là j'ai profité. C'était plus facile, j'avais moins de présence au bar. J'étais plus disponible qu'elle, j'avais plus de créneaux, j'étais plus accessible, c'était tout bénef pour lui et là être sans son papa, c'est dur, il [son fils] fait moins de choses, moins de tennis, moins de cinés. Avec sa sœur, on allait au parc, à la piscine, j'étais très proche. »

50Laurent représente l'exemple le plus manifeste des hommes classés dans la paternité brisée et qui ont bénéficié de visites. Le projet parental a du sens pour lui et s'est construit bien avant l'incarcération. Au cours des entretiens, il va à de nombreuses reprises comparer des événements passés aux instants présents vécus au cours de son incarcération.

\subsection{Le choc de l'incarcération : «on est là comme un con »}

- 26 De nombreux $\cdot$ ses auteur·e·s américain·e·s mettent en évidence deux raisons principales qui expliquen $\underline{(. . .)}$

51La paternité brisée se vit en prison comme une expérience aux effets destructeurs sur le lien père-enfant. La séparation avec l'enfant est présentée comme « une seconde peine » (Cardi, 2008, 289). Les hommes concernés souffrent de cette situation et se placent comme les victimes d'un système dont ils n'ont pas les clés. Le statut de détenu est vécu 
douloureusement et contraire au statut paternel par les enfants et par le père. Ces hommes s'évaluent négativement et ont le sentiment d'avoir chuté tant socialement que familialement. La remise en cause de leur rôle peut aller de la décision de ne pas voir les enfants pendant cette période au renoncement des enfants de venir en prison pour ne pas voir leur père en état de faiblesse. Selon la nature du lien conjugal, les mères ont plusieurs positions allant du soutien du père au refus de cette paternité incarcérée 26. Les hommes en couple (six sur les quatorze) bénéficient de parloirs, de contacts téléphoniques et de courriers réguliers avec les enfants. Cette relation est soutenue par la mère. Quant aux pères séparés, ils décrivent deux situations : dans la première, les mères refuseraient qu'ils voient les enfants et beaux-enfants, mais ne s'opposeraient pas aux contacts téléphoniques tout en ne favorisant pas les réponses à leurs courriers. Dans la seconde, les pères n'ont aucun contact téléphonique ou par courrier. Les mères refuseraient de leur donner des nouvelles et, dans le pire des cas, ils ignorent où se trouvent mères et enfants.

52Dans le cas de Laurent, pendant les deux premiers mois de sa peine, seule sa compagne lui rend visite car elle craint que les enfants ne soient traumatisés. Il n'a pas le droit de leur téléphoner par ordre du juge. Sa compagne accepte finalement de les lui emmener.

La première fois où j'ai vu mes enfants au parloir, je les ai pris direct dans mes bras, la deuxième fois j'ai pas pleuré et ma fille me l'a fait remarquer, car à Noël, ça faisait deux mois, j'ai craqué et eux aussi, c'était émotionnellement très fort. On a parlé de tout, de leur école, du copain de ma fille.

(Laurent, 38 ans, marié, deux enfants [14 ans et 17 ans], incarcéré depuis 8 mois)

53Au cours du parloir, Laurent discute longuement avec ses enfants, cependant il fait souvent le parallèle entre ce qu'il vivait et faisait avec eux avant la prison et ce qu'il ne peut plus faire.

Ils m'en veulent d'être en prison, mon fils me l'a écrit : «je fais moins ceci moins cela, je t'en veux ». Mon absence a changé le quotidien de mon fils, $\mathrm{j}$ 'étais plus présent que sa mère, on allait au tennis, j'étais toujours avec lui quand je ne travaillais pas, je ne faisais pas de trafic. Mon temps libre, il était pour mes enfants, donc voilà. [...] J'ai vraiment de magnifiques souvenirs avec eux. [...] Je pense à tous ces moments quand je suis seul dans ma cellule et c'est dur de penser à tout, de savoir qu'on est là comme un con. [...] C'est important pour moi d'élever ses enfants... on ne rigole pas avec l'éducation. Il faut être là dès le départ, c'est pour ça que je le vis très mal. Quand je n'étais pas en prison, j'attachais beaucoup d'importance à ça, j'étais derrière eux. Au quotidien, je les ai accompagnés pour qu'ils soient heureux, équilibrés, qu'ils ne manquent de rien et pas que matériellement et ça c'est... C'est pour ça que je suis malheureux, c'est hyper douloureux. »

(Laurent, 38 ans, marié, deux enfants [14 ans et 17 ans], incarcéré depuis 8 mois)

- 27 Cette mise en récit de la « vie ordinaire » se retrouve chez la « criminelle conforme » de Cardi $(2$ (...)

- 28 Marie Douris et Pascal Roman $(2014,141)$ expliquent que la situation de détention provoque l'invers (...)

54Laurent a deux enfants plus âgés, ce qui n'est pas sans effet sur le sens de sa paternité et sa capacité à l'analyser. Il a « fait » le père, en expérimentant différentes phases parentales 27 . Il craint de perdre le lien qu'il a tissé avec ses enfants et éprouve une culpabilité d'être en 
prison. L'incarcération conduit à une inversion générationnelle 28 où le père doit être pardonné pour ses fautes. L'extrait sur le décalage entre ce qu'il vit au parloir et ce qu'il vivait à l'extérieur met en évidence l'impact de l'incarcération sur le quotidien de ses enfants (Justin Dyer 2005 ; Hairston, 1998, 1995).

55La paternité brisée s'inscrit dans une insatisfaction parentale face à laquelle les dispositifs comme le parloir se révèlent inadéquats, en comparaison à la relation passée.

- Ma fille est revenue en février avec mon fils, tous les deux en même temps à ma grande surprise générale. Et puis ma fille est plus revenue, mon fils aussi, ils ont dit à leur mère qu'ils ne voulaient plus venir.

—C'était trop dur?

- Oui.

— Pour quelles raisons ?

- C'est le contexte, l'ambiance, c'est me voir impuissant, ils savent que je ne suis pas bien, je peux pas montrer que je suis joyeux. Le contexte, l'endroit, c'est lugubre, crado, ce n'est pas le top. Tu vois en 2010 les parloirs étaient comme ce bureau, y avait pas de séparation, à Fresnes c'est pas ça. Ça fait $2 \mathrm{~m}^{2}$, y a un muret avec un petit comptoir c'est pas... ça craint.

(Laurent, 38 ans, marié, deux enfants [14 ans et 17 ans], incarcéré depuis 8 mois)

56Pour Laurent, les conditions du parloir sont peu favorables. Il peut difficilement communiquer sa joie de retrouver ses enfants tant sa condition physique - être incarcéré et matérielle s'impose à la rencontre. La perception du lieu influence l'image que Laurent a de lui-même. Le poids de l'institution conduit parfois les enfants à renoncer à ces visites en dépit de l'attachement affectif éprouvé pour leur père. Dans le cas de Laurent, la visite au parloir confronte ses enfants à la situation de leur père : il est en prison, diminué, dominé (Chantraine, 2004, 230) et impuissant. Cet exemple montre comment le rôle paternel est ébranlé. Laurent, comme les détenus étudiés par Creasie Finney Hairston (1998), se préoccupe de l'image paternelle que ses enfants perçoivent.

\subsection{Un sentiment d'illégitimité du rôle paternel : «c'est douloureux, je suis passé à côté »}

57Les pères concernés par la paternité brisée se trouvent généralement dans un état d'abattement. Peter Burke $(1991,74)$ atteste que l'interruption ou la modification de l'identité paternelle provoque une plus grande détresse pour la personne qui se définit comme très engagée. Cette détresse n'est de fait pas vécue de la même manière selon les individus. Tous les pères rencontrés éprouvent à un moment de leur incarcération un certain mal-être cependant ce sentiment semble exacerbé chez les détenus regroupés dans ce type, car ils évoquent de manière riche leur passé parental avant l'incarcération.

58L'incarcération déstabilise et les comportements attendus sont modifiés. Les visites ou leurs absences conduisent à un travail d'évaluation des pertes qui les obligent à ajuster leur rôle aux contraintes carcérales. La mise en présence du détenu et de ses proches conduits à 
une transposition des attentes de chacun des partis, mais les récits mettent en exergue les possibilités restreintes d'actions dans ce lieu (discussions, jeux et quelques contacts physiques) spatialement et temporellement. Les pratiques parentales expérimentées avant la détention ne peuvent plus être actualisées. Christine Dufourcq-Chappaz $(2011,150)$ se réfère au terme psychologique de « deuil du père idéal ». L'un des aspects de la paternité brisée se définit par le sentiment d'illégitimité du rôle paternel. Laurent tente de maintenir son autorité en cachant le motif de son délit.

- Mon fils ne sait pas encore vraiment la cause, il tomberait du haut de l'armoire. Moi qui lui parle de valeurs et tout, qui fais du sport, il tomberait des nues.

— Tu as peur que ça impacte ton autorité ?

- Oui je pense, je perdrais pas mal de crédibilité et il serait déçu, je pense que voilà je suis son papa avant tout. Il m'idolâtre, il est fier de moi, il prend exemple sur moi, ça le décevrait beaucoup.

\section{— Et tu feras comment?}

- Je l'évoquerai, je vais attendre qu'il soit plus mature, quand le moment sera venu. Je ne vais pas précipiter les choses. Je vais pas me dire « il faut que je lui dise », je lui dirais quand le moment sera venu, mais pas tout de suite.

(Laurent, 38 ans, marié, deux enfants [14 ans et 17 ans], incarcéré depuis 8 mois)

- 29 Laurent explique les difficultés de son fils à l'école dues à des troubles du comportement, qui jus (...)

59Le fils de Laurent pense qu'il est incarcéré à cause d'une bagarre. Par ce non-dit, l'enquêté tente de préserver l'image qu'il donne à son enfant. L'incarcération engendre chez lui un sentiment de honte et de faute. Laurent se persuade que la vérité entraînerait inévitablement la perte de son autorité paternelle. La peur d'être considéré comme un « mauvais père » et d'être confronté à la décrédibilisation de son autorité le pousse à évoquer son incarcération par petites touches. Certains détenus « [cultivent] la nuance : [révélant] la vérité en fardant le détail troublant, pécher par omission [pour] négocier le mensonge » (Rambourg, 2006, 57). Pour Marie Douris et Pascal Roman $(2014,141)$ « dire la réalité de son incarcération représente pour l'individu détenu le degré de responsabilisation le plus aigu de ses actes à l'égard d'autrui », mais incorporer la prison à l'organisation familiale s'avère délicat. Beaucoup de détenus espèrent que les jeunes enfants oublieront cette expérience ou reportent le récit de l'incarcération à la sortie. L'accès à la vérité n'est pas toujours équitablement réparti entre les enfants. La fille de Laurent connaît les raisons de son incarcération et ils ont échangé de longues lettres à ce sujet. L'âge de son fils peut justifier ce choix, mais la nature du lien les distingue aussizi .

60La paternité brisée se définit également par la crainte de perpétuer un modèle familial défaillant. Avoir des enfants semble être le moyen de «donner ce qu'on n'a pas eu »expression récurrente dans les entretiens -, mais l'incarcération matérialise l'échec de cet idéal. J'illustrerai mon propos avec un extrait de Laurent : 
Quand j'ai eu des enfants, je ne voulais pas qu'ils subissent, je voulais donner du bonheur comme je n'ai pas eu et utilisé mon expérience pour que ça leur soit bénéfique. Tout ça, c'est douloureux, je suis passé à côté, j'ai voulu apporter du bien et là incident de parcours, c'est un gros dérapage, c'est pas bon pour leur équilibre, je l'ai mauvaise, j'ai les boules, ça fait du mal pour mon cœur, j'ai loupé mon truc, ce qu'il y a de plus cher à mes yeux, l'éducation de mes enfants.

(Laurent, 38 ans, marié, deux enfants [14 ans et 17 ans], incarcéré depuis 8 mois)

- 30 Les relations entre les détenus et leurs parents sont fragiles. Les enquêtés décrivent souvent des (...)

61La plupart des détenus craignent de reproduire le modèle de paternité30 qu'ils ont connu. L'arrivée de leur enfant les encourage à une réflexion propice à la construction de leur propre destinée de père : " plutôt que de se voir condamnés à reproduire la pauvreté du modèle paternel reçu en héritage » (Ouellet et al., 2006, 165), ils vont chercher à fabriquer le leur. Les enquêtés passent ainsi d'un discours très construit de ce que fait un père selon eux (conduire les enfants à l'école et dans leurs activités, être à l'écoute, gronder, prendre en charge certaines tâches quotidiennes) à un sentiment de découragement.

62Les descriptions peuvent interroger l'idéalisation des relations en prison : il est vrai qu'en contexte de disqualification sociale, la paternité devient une position valorisante investie massivement dans les discours et parfois peu dans la pratique. Toutefois, la paternité brisée met en évidence des liens stables et une relation affective établie avant la détention. Les enfants des hommes regroupés dans la paternité brisée sont souvent plus âgés que ceux des pères classés dans la paternité marginale. Cette différence conduit les premières cités à avoir expérimenté plus longuement leur paternité et à étayer leur réflexivité paternelle. Les pères dont les enfants sont plus jeunes se sentent quant à eux privés — notamment par la mère de l'expérience d'être père. Les différents rôles parentaux (éducatif, affectif, autorité, financier) étaient investis et s'inscrivent dans l'histoire de ces hommes. Leur capacité à décrire précisément leur modèle paternel rend l'ajustement à la détention d'autant plus problématique qu'ils ne peuvent plus endosser ce rôle de manière satisfaisante. Ils déclarent avoir et vouloir assumer pleinement leur paternité. Ils expérimentent avant tout la paternité de manière négative et dans la souffrance.

\section{La paternité ressource}

63Dans l'article Fathers in Jails, Brad Tripp $(2009,39)$ montre que la dernière incarcération, « the last time » est régulièrement associée à l'identité paternelle. À la lecture de l'ouvrage collectif Les sorties de délinquance, il apparaît que « l'arrêt de la délinquance repose sur le fait que l'individu possède une motivation ou des perspectives suffisantes pour pouvoir se détourner de la délinquance » (Mohammed et al., 2012, 109). Par motivation ou perspectives, les auteur·e's entendent par là des «planches de salut» (par exemple le mariage, un emploi) qui pourront entraîner « une diminution de la désirabilité et de la pertinence d'un comportement délictueux ». La paternité ressource s'inscrit dans un projet de fin de la délinquance et une réflexion sur l'incarcération. La peine de prison — qu'elle soit la première ou non - est vue comme un moment de décision. Ces pères, à la différence des paternités brisées, objectivent les ressources - notamment familiales — dont ils peuvent se saisir pour impulser une bifurcation biographique (Bessin et al., 2009, 277). 


\subsection{Devenir père et le rester}

64Les détenus concernés par la paternité ressource sont au nombre de quinze. Ils ont une histoire caractérisée par un investissement précoce dans la délinquance ou par un motif d'incarcération lourd (homicide-mœurs) : la surreprésentativité des détenus incarcérés en centre de détention dans ce type peut s'expliquer par une stabilité plus grande qu'en maison d'arrêt (Chantraine, 2004, 42). Ils sont également incarcérés pour des peines supérieures à deux ans.

65Le projet de devenir père n'a pas été laissé au hasard et, pour certains, a été réalisé pendant l'incarcération. C'était à la fois un projet de couple, d'enfant, mais surtout de famille. La particularité de cette paternité est qu'elle s'inscrit dans une mobilisation des relations sociales autour du détenu pour maintenir un statut et un rôle qui lui sont reconnus au sein de la famille. Il peut à la fois « compter sur» sa famille et avoir le sentiment de « compter pour » eux. Je m'intéresserai à deux exemples qui illustrent le premier aspect de la paternité ressource : devenir père. Sofiane a 32 ans, il est marié depuis 9 ans quand il a son premier enfant. Devenir père s'inscrit dans le prolongement du couple. «J'ai fait les choses dans les règles par étapes, je me suis marié puis j'ai eu mes enfants, j'ai essayé de suivre ça ». Avoir des enfants l'a motivé à prendre moins de risques, mais sa paternité s'imbriquait avec son activité délictuelle. «Ça a été un coup de frein, mais pas aussi radical que maintenant. J'avais forcément mes enfants en tête, mais je prenais les risques, on sait ce qu'on peut perdre, mais le goût du risque ou de l'argent peuvent vous mettre un voile. Je me levais le matin, j'accompagnais mes enfants à l'école puis j'attendais un coup de fil et j'avais un rendez-vous. C'est un peu comme un travail, mais un travail malhonnête ». La paternité sans pour autant tout bouleverser esquisse un souhait de changement qui se prolonge au cours de la détention. C'est le cumul d'événements (paternité, incarcération, éloignement familial, prise de conscience) qui va amorcer une transformation. Les auteur.e.s de l'ouvrage Bifurcations : les sciences sociales face aux ruptures et à l'événement (Bessin et al., 2009) montrent que c'est la durée des événements et la place de ces derniers dans le parcours qui conduisent à penser l'avenir sur un autre mode.

66Timur est âgé de 34 ans. Il a un fils, Sayam, de 19 mois, conçu en parloir. Incarcéré depuis 7 ans pour homicide involontaire, il effectue une peine de 20 ans. Au cours de son incarcération, il engage une relation épistolaire avec son actuelle compagne qui par la suite a souhaité le rencontrer en parloir. Avant de s'engager avec elle, il attend trois ans, le temps d'obtenir le délibéré pour qu'elle puisse « savoir à quoi s'attendre ». Ils décident de faire un enfant, «j'allais avoir 36-38 ans à ma sortie et j'avais peur d'être trop âgé ». Il bénéficie de trois parloirs par semaine et d'UVF. Sa compagne a déménagé pour se rapprocher de la prison. Lors de nos entretiens, Timur explique avoir tué l'amant de son ex-compagne par excès de jalousie et des problèmes de toxicomanie. A posteriori, je découvre que le motif était autre : sa fille est décédée à la suite des nombreux coups qu'il lui a infligés en présence de la mère (elle-même incarcérée pour non-assistance à personne en danger), tous deux sous l'emprise de stupéfiants.

67Sofiane et Timur, en dépit d'une certaine précarité sociale — délinquance, expériences professionnelles rares et précaires, incarcération — sont devenus pères. Ils disent l'avoir souhaité et désiré. Le vécu conjoint de la paternité et de l'incarcération provoque un désir de rupture avec un mode de vie ou une trajectoire précaires. Ce statut parental acquiert un sens particulier qu'ils vont expérimenter pendant leur l'incarcération. 


\subsection{Expérimenter sa paternité en détention : « c'est un peu ma colonne vertébrale »}

68 Une des questions qui s'est posée au cours de cette enquête était de savoir dans quelle mesure la paternité se construit et prend sens au cours de la détention.

69Interroger les conditions de la paternité en prison donne accès à un ressenti au moment présent de l'entretien, construit à partir d'une réinterprétation du passé. Les pères que j'ai classés dans ce type investissent au maximum la relation père-enfant par le parloir, les UVF et le téléphone (parfois de manière illégale). Du fait de la détention, ces hommes réalisent la perte qu'ils subissent comme pères, ce qu'ils ne peuvent plus faire (être là au quotidien, le contact, l'autorité) ou qu'ils n'ont pu expérimenter. Ce sentiment va les amener à investir davantage le lien à leurs enfants pendant la période d'incarcération et cela grâce au soutien de leurs proches.

- 31 Les deux pères rencontrés qui bénéficient des Relais enfants-parents sont dans la paternité ressour (...)

70Pour les hommes en couple, il existe un dialogue important avec la mère, ils construisent ensemble une parentalité de couple ; la mère leur permet d'apprendre à être père (Touraut, 2014). Pour le cas de pères séparés, la mère ne s'oppose pas aux visites $\underline{31}$. Une solidarité parentale peut aussi se constituer pour prévenir la fragilisation, en aidant à amener les enfants au père.

- 32 L'usage du téléphone et du courrier devient central quand l'enfant est en mesure de l'utiliser et $\mathrm{s}(\ldots)$

71En m'interrogeant sur le vécu du parloir, je tente de comprendre ce qui se joue lors de cette mise en présence. Il existe d'autres moyens de soutenir la relation, mais à ses débuts, quand les enfants sont jeunes, il apparaît que pour se sentir père, l'expérimentation du lien est fondamentale 32 . Caroline Touraut $(2014,312)$ montre l'importance de la présence pour « certifier le lien ». Si elle se concentre davantage sur le lien conjugal, son analyse peut aisément se transposer à la parentalité. Les parloirs «sont présentés comme une nécessité affective autant qu'ils répondent à un besoin de réassurance ». Les entretiens révèlent ce manque de contact «peau contre peau ». Les détenus tentent de se réapproprier des gestes simples et démonstratifs de leur affection au cours de ces interactions. Le contact physique symbolise la figure d'attachement et encourage la formation, la reconstruction ou la restructuration de l'identité paternelle. Ces retrouvailles marquent une consolidation des liens affectifs et renseignent les détenus sur la place conservée auprès de leurs enfants.

72Concernant le déroulement des parloirs, Sofiane expose les difficultés auxquelles est confrontée sa compagne :

C'est horrible pour ma femme. Se lever tôt le matin, attendre $3 / 4$ d'heure puis encore après. C'est très dur pour une femme de faire ça une fois par semaine, ce n'est pas évident pour elle à l'extérieur pourtant je lui ai dit de ne venir qu'une fois par mois. Elle a décidé autrement pour qu'il y ait un suivi. La plupart du temps, elle vient au parloir avec les gosses, mais quand on doit discuter sérieux, elle vient seule. 
(Sofiane, 32 ans, marié, 2 enfants [3 ans et 5 ans], incarcéré depuis 20 mois)

73Sofiane est conscient des coûts (temporels, physique) du déplacement pour venir le visiter. Il tente de dissuader sa compagne de venir aussi fréquemment mais, par les visites, elle souhaite maintenir sa place dans le réseau familial ; le suivi, pour être efficient, doit être régulier. Elle joue un rôle actif pour entretenir l'implication et la prise de décision de Sofiane au quotidien. L'affirmation de Marie Douris et Pascal Roman $(2014,139)$, selon laquelle « il n'y a pas de parentalité sans conjugalité », trouve un écho dans cette enquête. Le parloir devient fondamental pour perpétuer un rôle paternel.

- En parloir, je m'amuse beaucoup avec eux, j'essaye de rire et puis je parle avec leur mère, on rigole ensemble. C'est un moment ensemble. On essaye de rendre la misère agréable [...].

— Tu préférerais ne pas les voir?

— Je n'irais pas jusqu'à dire ça, à chaque fois que je les vois, c'est une dose d'énergie positive, c'est vraiment réel. Et quand on sort du parloir, qu'il y a quelque chose qui s'est passé, on les a pris dans ces bras, il se dégage quelque chose [...], c'est un peu ma colonne vertébrale.

(Sofiane, 32 ans, marié, 2 enfants [3 ans et 5 ans], incarcéré depuis 20 mois)

- 33 Marc Bessin et al. $(2009,282)$ expliquent également que pour bifurquer de manière positive, quatre $(. .$.

74L'importance de la famille s'exprime à la fois dans la sortie de la délinquance et lors des visites : toutes deux introduisent une dynamique sociale dans la mesure où elles apportent « de nouvelles motivations, ainsi qu'une dynamique psychologique de réassurance et d'estime de soi » (Mohammed et al., 2012, 205)33. Pour Sofiane, chaque visite réintroduit du réel. Le jeu est un des moyens de renouer le lien. Avec des enfants en bas âge, le parloir doit être un moment distrayant qui laisse un bon souvenir ; le but est d'oublier le contexte pesant de la détention. La rencontre permet d'instaurer un moment « comme avant » et de retrouver des sensations qui ne soient pas exclusivement subordonnées à la vie en détention. C'est un soutien psychologique et moral qui permet d'éviter « la dépersonnalisation et la déréalité carcérale » (Touraut, 2012).

75Pour Timur, qui est devenu père en prison, les visites deviennent essentielles à la (re) construction de sa paternité. Le parloir devient un lieu d'apprentissage et constitue un support relationnel tourné essentiellement vers le jeu. Durant ce temps court, la relation à l'enfant devient prioritaire. Le couple est souvent placé entre parenthèses.

\section{— Et les UVF c'est comment?}

- Ça va de mieux en mieux, au début il marchait pas, je lui changeais la couche, je le lavais, maintenant il marche. Le dernier UVF, il commençait à marcher et il va partout, il joue avec son père, avec sa mère. Et il est content. Il est excité. Dès qu'il me voit, il est excité. Sa mère lui parle beaucoup de moi [...]. Au moins, je peux manger avec lui, dans les parloirs on ne peut pas manger, on ne peut pas ramener de bonbons. Ils nous prennent la tête pour rien. Mon fils, à chaque fois, il fouille dans mes poches. Je prends des risques pour lui comme ça il sait que papa lui donne à manger. [En UVF] Je fais le rôle du père et de la mère, vu que j'ai pas 
beaucoup l'occasion de m'occuper de lui. Je vais le voir quand il pleure. On va sur le balcon, même quand je fume. On regarde des films. Il aime bien que je lui chante Ainsi font font font les petites marionnettes, Frère Jacques, il danse, il claque des mains, il est content.

— Il y a une différence selon toi entre une mère et un père ?

— Non, c'est juste que vu qu'il voit souvent sa mère, c'est bien qu'il voit son père, qu'il s'habitue à moi.

(Timur, 34 ans, concubinage, 1 enfant [1 an], incarcéré depuis 7 ans)

76Contrairement au parloir, considéré comme contraignant, les UVF permettent de disposer d'une relative liberté de mouvement (Lancelevée, 2011 ; Rambourg, 2006). Timur souligne cette différence avec l'épisode des bonbons. L'impossibilité de partager un repas est un exemple récurrent pour les détenus. Il constitue un support non négligeable dans la relation.

77Les UVF, en tant que lieu de vie partagé, se rapprochent du monde extérieur dans leur déroulement. La reconstruction d'un espace intime encourage l'investissement des rôles, particulièrement limité en parloir. Timur, à travers une forme de quotidienneté, expérimente et acquiert des compétences parentales. Il veut tout faire et ne rien manquer. Francine Allard et Lise Binet (2002), qui s'intéressent à l'engagement parental des pères en situation de pauvreté, montrent que ce sentiment de compétence s'acquiert justement par des actions directes et des expériences vécues positivement.

\subsection{Un futur après la détention : « depuis que j’ai mon fils, je pense à la sortie»}

78La paternité ressource s'expérimente en prison, mais elle donne aussi du sens à la peine, tout en permettant d'envisager un futur après la détention. Ces hommes se sentent soutenus : avant d'être détenus, ils sont pères. Le réseau de solidarité qui se tisse autour d'eux créé par la mère et la famille leur permet d'engager une réflexion sur le sens de paternité.

- 34 «L'importance de l'événement est associée au regard subjectif que cet événement provoque pour les $\underline{(\ldots)}$

79J'ai évoqué l'aspect d'une réparation, voire d'une bifurcation biographique, liée à l'arrivée de l'enfant $\underline{34}$ en détention. Timur analyse ce qu'il considère comme une dernière chance :

— Qu'est-ce qui a changé depuis la naissance de ton fils?

- 35 Quelques détenus refusent la «gamelle » servie par l'administration pénitentiaire, dénonçant la ma (...)

— Ça fait un moment que je suis là, je me rends compte de plusieurs choses. Depuis que j'ai mon fils, je pense à la sortie. Avant je m'en foutais, un an, six mois c'était pas grave, même vingt ans. Là je travaille, je demande jamais de sous. Je veux qu'ils soient bien même si je prends la gamelle 35 , vaut mieux qu'eux soient bien. [...] J'ai la pêche, je sais que j'ai un garçon et une femme qui m'attendent, mais c'est plus dur, on se fait beaucoup de soucis pour les gens qui sont dehors. On se pose beaucoup de questions [...] Je prépare tout doucement la 
sortie [...] C'est facile d'imaginer en prison son futur, mais faut que ça se réalise, je suis un bon cuistot, j'aimerais bien ouvrir mon resto, je suis d'origine turque, j'avais un Kebab. Mon but c'est de les rendre heureux, car le temps que j'ai passé ici, elle a été là pour moi, je veux les emmener en vacances, qu'ils ne se plaignent de rien, qu'ils ne manquent de rien. Je veux sortir avec un travail et pendant que je m'occuperai de mon resto, je pourrai être présent. Fini de traîner et guetter la cage [prison]. Je suis arrivé à 27 ans, j'ai 34 ans, je vais sortir à 37 ans, c'est fini tout ça, ça va être ma famille avant tout.

(Timur, 34 ans, concubinage, 1 enfant [1 an], incarcéré depuis 7 ans)

- 36 Dans l'ouvrage de Mohammed et al. (2012), de nombreux chercheurs ont montré que le choix et la déci (...)

80Les effets concomitants du vieillissement, de l'entrée dans la paternité et de la détention provoquent une prise de conscience de Timur sur ses choix et ses actes. Il réfléchit à la manière d'être un père tout en imaginant les événements positifs qui surviendront après la libération $\underline{36}$. Certains détenus adoptent souvent un discours fantasmatique qui leur permet de se maintenir, se stimuler et d'écarter l'ombre des espoirs déçus. Timur souligne à juste titre la facilité d'imaginer son futur en prison, mais ce futur n'a de sens que s'il est transformé en avenir (Marchetti, 2000, 266). C'est grâce à des événements ponctuels qu'il arrive à décrire son rôle de père :

\section{— Tu dirais que tu es comment comme papa?}

— Je suis un père câlin, ça c'est sûr, vu le nombre de câlins que je lui fais, ma copine elle en peut plus, elle est jalouse. Quand elle vient sur moi, il vient, il la pousse pour venir sur moi. Dès que je lui fais un bisou, il veut pas, il dit «non non ». Je dirais que je suis un père câlin, généreux, car franchement je lui envoie beaucoup, je ne dépense pas beaucoup pour moi, je suis fier de moi, si je sais qu'il manque, je me sens plus mal que si c'est moi qui suis dans la galère. Je suis un père, je suis là, oui je l'aime. Je lui donnerais ma vie, mais j'aimerais travailler, ramener de l'argent, je sais que c'est dur, je connais des familles qui ont du mal à finir les fins de mois. Elle a une paye au début du mois et moi je complète la fin. J'essaye de faire mon rôle de père même si je ne suis pas là. C'est facile de dire que je suis un père, car on n'est pas là, on sert à rien, ici je ne sers pas à grand-chose. [...] Moralement par rapport à ma peine, je me dis que c'est bien d'avoir un enfant, mais d'un côté, je pense à eux, je m'inquiète, j'aimerais être là.

(Timur, 34 ans, concubinage, 1 enfant [1 an], incarcéré depuis 7 ans)

81Timur se décrit comme un père aimant et investi financièrement. En dépit de l'incarcération, il montre son autonomie, son dynamisme et sa capacité à soutenir sa compagne. Il privilégie leur bien-être au sien. Â la lumière de cet extrait et de la définition de l'engagement paternel de Lamb et al. (1987), de nombreuses dimensions de la paternité émergent : la présence physique et la prise en charge de tâches et de responsabilités relatives à l'enfant, un soutien affectif, des interactions père-enfant significatives ainsi qu'une contribution financière et matérielle. Le plaisir ressenti au contact de l'enfant et sa réciprocité permettent la construction d'un rôle fort et affirmé. Timur identifie les compétences qu'il a acquises tout en soulignant ses réussites et ses manques. Être pourvoyeur demeure une dimension valorisée, mais qui n'est pas suffisante pour faire de lui un père. L'engagement paternel est décrit comme pluriel et découle d'un long processus qui passe par 
l'expérimentation du lien père-enfant. La reconnaissance par la mère, « elle lui parle de moi » renforce son existence en tant que père et son sentiment de compétence.

82Pour subsister, la paternité suppose une solidarité familiale forte. Elle fonctionne également comme support identitaire et relationnel. Précédemment, Sofiane décrivait sa famille comme une colonne vertébrale : les liens ont comme fonction première d'assurer un maintien identitaire et moral.

- C'est un soutien de les avoir, ça me permet de ne pas me sentir abandonné, si c'était le contraire je me sentirais abandonné. Je suis bien content que ça se passe comme ça, je connais des cas complètement différents où les mecs se tapent la tête contre le mur. [...] Être père, ça fait partie de ma vie, de mes choix de vie, je me suis retrouvé en prison, je ne l'ai pas voulu, mais je l'ai cherché. Je le vis bien, je sais que j'ai encore une carte à jouer dehors, j'ai quand même un pas d'avance, j'ai ma famille, maintenant faut plus que je me perde, pas de récidive, pas d'histoires qui en valent pas la peine.

- Y a une prise de conscience ?

— J'ai l'impression, enfin ce n'est pas le système de la prison ou d'avoir peur, c'est une prise de conscience sur le simple fait de vouloir réussir sa vie et leur donner un avenir, une vie normale.

(Sofiane, 32 ans, marié, 2 enfants [3 ans et 5 ans], incarcéré depuis 20 mois)

83Cet extrait confirme l'importance des visites pour « tenir » en détention ; en recevoir fait la différence. La responsabilité d'être père n'est pas seulement le fait de prendre un engagement, mais surtout de le respecter (Gaudet, 2001) dans un temps long. La paternité et la famille deviennent un atout qu'il ne faut pas gâcher. Le discours de Sofiane permet d'identifier deux événements qui n'ont pas la même signification dans son parcours : la paternité est irréversible, ce qui n'est pas le cas pour l'incarcération. Il distingue le statut de détenu et celui de père.

— Être père ça existe, mais pas père détenu, sur la pièce d'identité y a écrit père, [la détention] c'est juste un temps et vu que je veux plus revenir, j'espère que ce sera fini.

—C'est quoi être père pour toi ?

— C'est tenir un engagement du premier jour de la naissance de l'enfant à... allez, je vais dire jusqu'à la majorité. La définition pour moi du père c'est quand tu deviens un homme, mais la vie est tellement longue que devenir un homme, c'est dur. Un homme c'est quelqu'un qui protège sa famille, ses enfants, sa femme et quelque part j'ai l'impression d'avoir échoué, du fait que je suis pas présent dans mon foyer.

(Sofiane, 32 ans, marié, 2 enfants [3 ans et 5 ans], incarcéré depuis 20 mois)

84Le statut de père prend-il le pas sur le statut de détenu ? Pour Sofiane, être détenu est une erreur de parcours qui se circonscrit à un temps donné défini par la peine, tandis qu'être père est un statut à vie qui se définit comme un choix personnel. Pour la paternité ressource, le statut paternel devient garant d'une identité sociale légitime (Martial, 2013) qui peut mettre à 
distance les échecs multiples (professionnels, familiaux, etc.), mais aussi être un catalyseur de projet, notamment après la sortie.

- 37 Les détenus de ce type travaillent en détention ou tentent d'avoir un emploi et des activités ( $\operatorname{scol} \underline{(\ldots)}$

85La paternité ressource se caractérise par une mise à profit du temps de l'incarcération pour amorcer une bifurcation biographique. J'ai décrit cette mise à profit à travers la paternité, mais elle s'inscrit de manière plus générale 37 . Il est possible que cette bifurcation échoue, mais l'objectif de cette recherche est d'analyser la réalité des détenus et comment ils la perçoivent. À la différence de la paternité brisée, les détenus tiennent un discours positif concernant leur présent et leur avenir, lié en partie aux solidarités familiales dont ils bénéficient.

\section{$\underline{\text { 6. Conclusion }}$}

86L'institution carcérale encadre les liens familiaux en déterminant les lieux et les moments où ils peuvent se vivre et s'exprimer. Dans ce contexte, la paternité des détenus pourrait être pensée comme homogène. Cependant, en s'intéressant aux trois éléments principaux que sont la biographie individuelle, le moment de l'incarcération et l'appropriation personnelle des normes de parentalité, quatre types de paternités ont émergé. La paternité marginale se caractérise par une entrée précoce dans la parentalité. En détention, le mode prison l'emporte sur le rôle paternel. La paternité suspendue se définit par l'interruption - temporaire - de la relation parentale, renvoyée à un futur hypothétique après la prison. La paternité brisée témoigne d'une ambition parentale non réalisée à cause de l'incarcération et d'un fort sentiment de disqualification. Enfin, la paternité ressource s'appuie sur une solidarité familiale forte où l'enfant est l'un des catalyseurs de projet (recherche d'emploi et de formation, stabilité conjugale, etc.) en détention et pour la sortie future. Les types de paternité, loin d'être figés, nous renseignent sur les pratiques et les représentations parentales selon les événements biographiques.

87Comme pour les détenus, les pratiques professionnelles et les représentations parentales ne sont pas homogènes au sein de l'institution carcérale. Deux raisons principales peuvent l'expliquer : tout d'abord, plusieurs corps de métier coexistent en détention et se différencient par leurs missions ; de plus, il n'existe pas de coordination nationale concernant la prise en charge de la question parentale en détention. La paternité, sans être inexistante, se situe aux confins de l'institution carcérale. Bien que l'étude ne porte pas spécifiquement sur les professionnel·le's, elle permet d'esquisser quatre tendances qui peuvent faire écho aux quatre paternités présentées. Pour la première, les professionnel·le·s considèrent la paternité dans sa fonction utilitaire pour les détenus (obtention de remises de peines et de permission, pression sur la compagne, etc.) et sous l'angle de l'incompétence parentale. Pour la seconde, les professionnel·le·s minorent la question paternelle au profit de dimensions qu'ils/elles jugent prioritaires (emploi, logement, exécution de la peine). Pour la troisième, la paternité est avant tout appréhendée sous l'angle de la reproduction d'un modèle familial défaillant, tout en considérant que les détenus sont responsables de leur situation. Enfin, pour la quatrième, les professionnel·le·s reconnaissent l'importance de la paternité pour les détenus et la conçoivent comme une ressource concrète pour la réinsertion future.

\section{$\underline{\text { Haut de page }}$}




\section{Bibliographie}

Des DOI sont automatiquement ajoutés aux références par Bilbo, l'outil d'annotation bibliographique d'OpenEdition.

Les utilisateurs des institutions qui sont abonnées à un des programmes freemium d'OpenEdition peuvent télécharger les références bibliographiques pour lequelles Bilbo a trouvé un DOI.

Allard F., Binet L., 2002, Comment des pères en situation de pauvreté s'engagent-ils envers leur jeune enfant? : Étude exploratoire qualitative, Beauport, Régie régionale de la santé et des services de Québec, Direction de santé publique.

Arditti J.A., Smock S.A., Parkman T.S., 2005, "It's Been Hard to Be a Father": A Qualitative Exploration of Incarcerated Fatherhood, Fathering, 3, 3, 267-288.

Bessin M., Bidart C., Grossetti M. (Eds.), 2009, Bifurcations : les sciences sociales face aux ruptures et à l'événement, Paris, La Découverte.

Bonvalet C., Lelièvre E. (dir.), 2012, De la famille à l'entourage : l'enquête Biographies et entourage, Paris, Ined éditions Grandes enquêtes.

Bouchard G., 2007, Vivre avec la prison : Des familles face à l'incarcération d'un proche, Paris, L'Harmattan.

Burke P.J., 1991, Identity Processes and Social Stress, American Sociological Review, 56, 6, 836-849.

DOI : $\underline{10.2307 / 2096259}$

Cardi C., 2008, La déviance des femmes. Délinquantes et mauvaises mères : entre prison, justice et travail social, thèse de sociologie sous la direction de Murard N., Paris, Denis Diderot Paris 7.

Cardi C., 2014, Les quartiers mères-enfants : l' « autre côté » du dedans: Protection et surveillance des mères en prison de femmes, Champ pénal/Penal field, XI, [en ligne] https://journals.openedition.org/champpenal/8762, DOI 10.4000/champpenal.8762.

DOI : $10.4000 /$ champpenal.8762

Cardi C., Abdallah S.L., 2014, Vécus de la carcéralité des mères et des pères, Champ pénal/Penal field, XI, [en ligne] https://journals.openedition.org/champpenal/8815, DOI 10.4000/champpenal.8815.

DOI : $10.4000 /$ champpenal.8815

Carlson B.E., Cervera N., 1991, Inmates and their Families Conjugal Visits, Family Contact, and Family Functioning, Criminal Justice and Behavior, 18, 3, 318-331.

Cassan F., Mary-Portas F.-L., 2002, Précocité et instabilité familiale des hommes détenus, Insee première, 828, [en ligne] http://prison.eu.org/IMG/pdf/doc-625.pdf.

Cassan F.,Toulemon L., 2000, L'histoire familiale des hommes détenus, Insee première, 706, [en ligne] http://www.epsilon.insee.fr/jspui/bitstream/1/598/1/ip706.pdf. 
Chantraine G., 2004, Par-delà les murs : Expériences et trajectoires en maison d'arrêt, Paris, Puf.

Désesquelles A., Kensey A., 2006, Les détenus et leur famille : des liens presque toujours maintenus mais parfois très distendus, Données sociales, La société française, 59-67.

Douris M., Roman P., 2014, Liens Familiaux et détention - $1^{\text {re }}$ partie : Comment être parent en prison, Ucly, Unil.

Dufourcq-Chappaz C., 2011, Être père malgré tout : Univers carcéral et parentalité, Lyon, Chronique Sociale.

Elias N., 1997, Du temps, Paris, Fayard.

Galland O., 2011, Sociologie de la jeunesse, Paris, Armand Colin [5 $5^{\mathrm{e}}$ édition].

DOI : $\underline{10.3917 / \text { arco.galla.2011.01 }}$

Gaudet S., 2001, La responsabilité dans les débuts de l'âge adulte, Lien social et Politiques, 46,71 .

DOI : $\underline{10.7202 / 000324 a r}$

Goffman E., 1970, Asiles - études sur les conditions sociales des malades mentaux et autres reclus, Paris, Les éditions de minuit.

Goyette M., Pontbriand A., Bellot C., 2012, Les transitions à la vie adulte des jeunes en difficulté : Concepts, figures et pratiques, Québec, Presses de l’Université du Québec.

Hairston F.C., 1995, Fathers in Prisons: Responsible Fatherhood and Responsible Public Policies, in Lexington Gabel K., Johnston D. (Eds.), Children of incarcerated parents, MA, Lexington Books, 21-26.

Hairston F.C., 1998, The forgotten parent: Understanding the forces that influence incarcerated fathers' relationships with their children, Child welfare, 77, 5, 617-637.

Hamel C., 2003, L'intrication des rapports sociaux de sexe, de race, d'âge et de classe : ses effets sur la gestion des risques d'infection par le VIH chez les français descendants de migrants du Maghreb, thèse sous la direction de Handman M.-H., Paris, EHESS.

Insee, 2002, L'histoire familiale des hommes détenus, Paris, Synthèses/Institut national de la statistique et des études économiques.

Joël M., 2017, La sexualité en prison de femmes, Paris, Presses de sciences po.

Justin Dyer W., 2005, Prison, Fathers, and Identity: A Theory of How Incarceration Affects Men's Paternal Identity, Fathering, 3, 3, 201-219.

Justin Dyer W., Pleck J.H., McBride B.A., 2012, Imprisoned Fathers and Their Family Relationships: A 40-Year Review From a Multi-Theory View, Journal of Family Theory \& Review, 4, 1, 20-47.

DOI : $10.1111 / \mathrm{j} .1756-2589.2011 .00111 . \mathrm{x}$ 
Kaufmann J.-C., 2011, L'entretien compréhensif - L'enquête et ses méthodes, Paris, Armand Colin [ $3^{\mathrm{e}}$ édition].

Lamb M., Pleck J., Charnov E., Levine J., 1987, A biosocial perspective on paternal care and involvement, Biology Faculty \& Staff Publications, 11-143.

Lancelevée C., 2011, Une sexualité à l'étroit. Les unités de visite familiale et la réorganisation carcérale de l'intime, Sociétés contemporaines, 83, 3, 107.

DOI : $10.3917 /$ soco.083.0107

Le Pape M.-C., 2005, Mémoire familiale, filiation et parentalité en milieux populaires, Recherches et prévisions, 82, 1, 17-32.

Marchetti A.-M., 2000, Perpétuités : le temps infini des longues peines, Paris, Plon.

Martial A., 2009, Le travail parental : du côté des pères séparés et divorcés, Informations sociales, 154, 96-104.

Martial A., 2012, Paternités contemporaines et nouvelles trajectoires familiales, Ethnologie française, 42, 1, 105-116.

DOI : $\underline{10.3917 / \text { ethn.121.0105 }}$

Martial A., 2013, Une paternité réinventée ? Le vécu parental des pères isolés, Informations sociales, 2, 62-69.

Mohammed M. (dir.), 2012, Les sorties de délinquance, Paris, La Découverte Recherches.

Nurse A.M., 2002, Fatherhood Arrested: Parenting from Within the Juvenile Justice System, Nashville, Vanderbilt University Press.

Ouellet F., Milcent M.-P.,Devault A., 2006, Jeunes pères vulnérables : Trajectoires de vie et paternité, Nouvelles pratiques sociales, 18, 2, 156.

Pailhé A., Mortelmans D., Castro T., Trilla C.C., Digoix M., Festy P., Krapf S., Kreyenfeld M., Lyssens-Danneboom V., Martín-García T., Rault W., Thévenon O., Toulemon L., 2013, Changes in the life course, Families And Societies, 67.

Quéniart A., 2008, La paternité sous observation : des changements, des résistances mais aussi des incertitudes, [en ligne]

http://classiques.uqac.ca/contemporains/queniart_anne/paternite_sous_observation/paternite_s ous_observation.pdf.

Rambourg C., 2006, Les unités de visites familiales, Cirap.

Régnier-Loilier A., 2013, Focus - Séparation et rupture des relations entre le père et l'enfant, Informations sociales, 2, 70-74.

Ricordeau G., 2008, Les détenus et leurs proches : Solidarités et sentiments à l'ombre des murs, Paris, Éditions Autrement. 
Schehr S., 1999, La vie quotidienne des jeunes chômeurs, Paris, Puf.

DOI : $10.3917 /$ puf.scheh.1999.01

Schultheis F., Frauenfelder A., Delay C., 2009, Les classes populaires aujourd'hui : portraits de familles, cadres sociologiques, Paris, L'Harmattan.

Schwartz O., 2012, Le monde privé des ouvriers, Paris, Puf.

DOI : $\underline{10.3917 / p u f . s c h w a .2012 .01}$

Touraut C., 2012, La famille à l'épreuve de la prison, Paris, Puf.

DOI : $10.3917 /$ puf.toura.2012.01

Touraut C., 2014, Parentalité partagée à distance : rôles parentaux et rapports conjugaux face à l'enfermement, Champ pénal/Penal field, XI, [en ligne]

https://journals.openedition.org/champpenal/8759, DOI 10.4000/champpenal.8759.

DOI : $\underline{10.4000 / \text { champpenal.8759 }}$

Tripp B., 2009, Fathers in Jail: Managing Dual Identities, Applied Psychology in Criminal Justice, 5, 1, 26-56.

Turcotte G., Dubeau D., Bolté C., Paquette D., 2001, Pourquoi certains pères sont-ils plus engagés que d'autres auprès de leurs enfants ? Une revue des déterminants de l'engagement paternel, Revue canadienne de psycho-éducation, 30, 1, 65-91.

$\underline{\text { Haut de page }}$

Annexe 


\begin{tabular}{|c|c|c|c|c|}
\hline \multicolumn{5}{|c|}{ 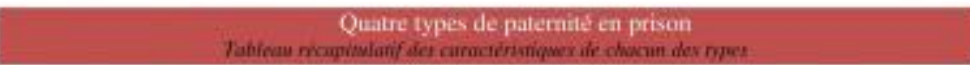 } \\
\hline & Paternité marsinale & Paterniaté suspendue & Paternité brisée. & Paternité ressource \\
\hline 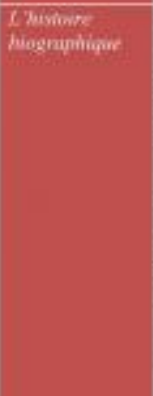 & 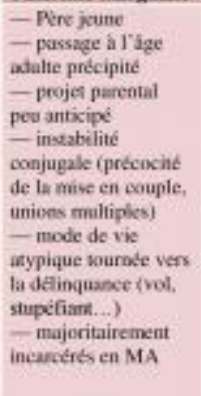 & 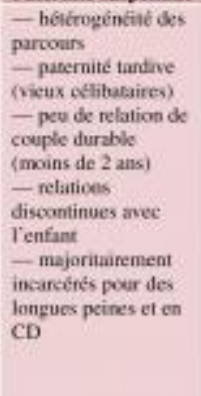 & 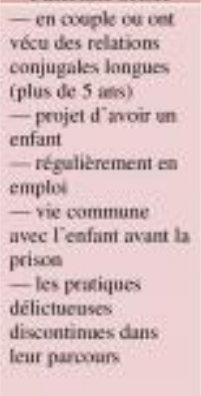 & 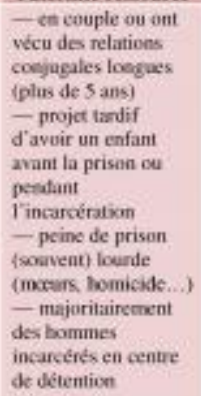 \\
\hline 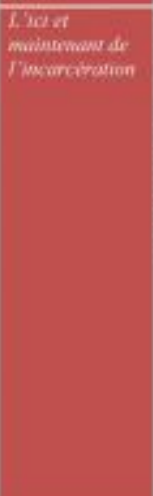 & 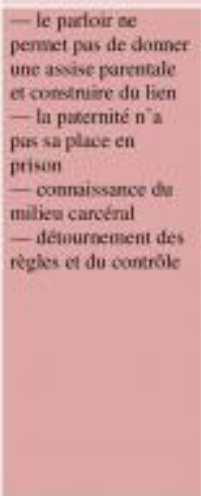 & 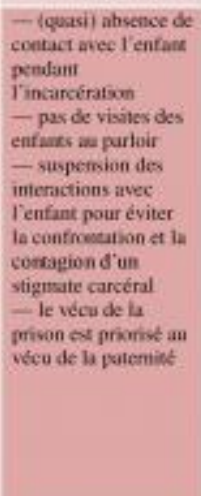 & 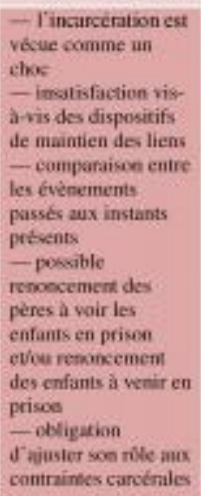 & 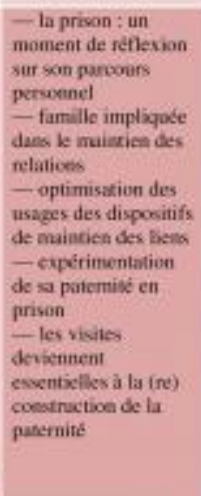 \\
\hline 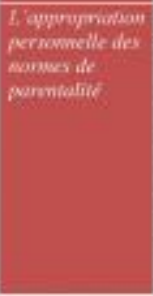 & 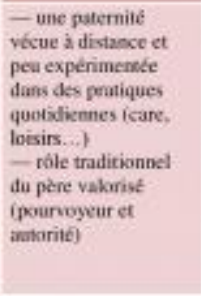 & $\begin{array}{l}\text { - discours pauvres } \\
\text { sur les protiques } \\
\text { paremales } \\
\text { - passivité dans ker } \\
\text { rük, remoyant la } \\
\text { responsahiline sar } \\
\text { lex-conjointe et sur } \\
\text { les contraintes } \\
\text { carcirales } \\
\text { - reprise des liens } \\
\text { renvayte à la sortie }\end{array}$ & 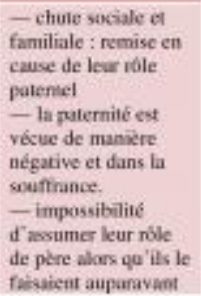 & 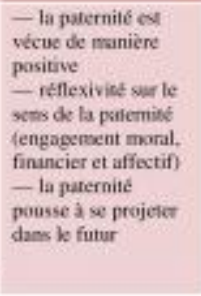 \\
\hline
\end{tabular}

$\underline{\text { Agrandir Original (jpeg, 724k) }}$

\section{Boxplots : quartiles par types de paternité}

\section{— médiane $\diamond$ moyenne $\cdot$ extrême}




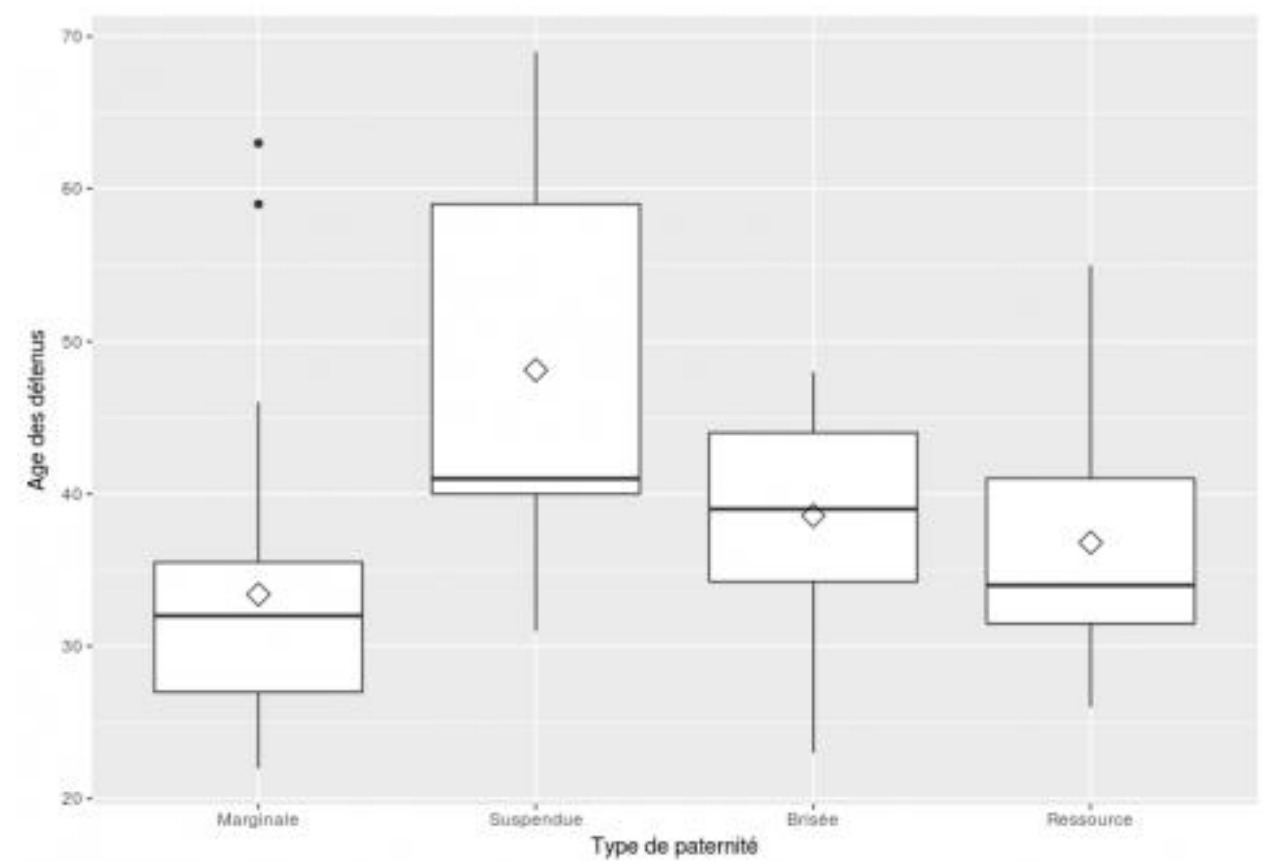

$\underline{\text { Agrandir Original (jpeg, 56k) }}$

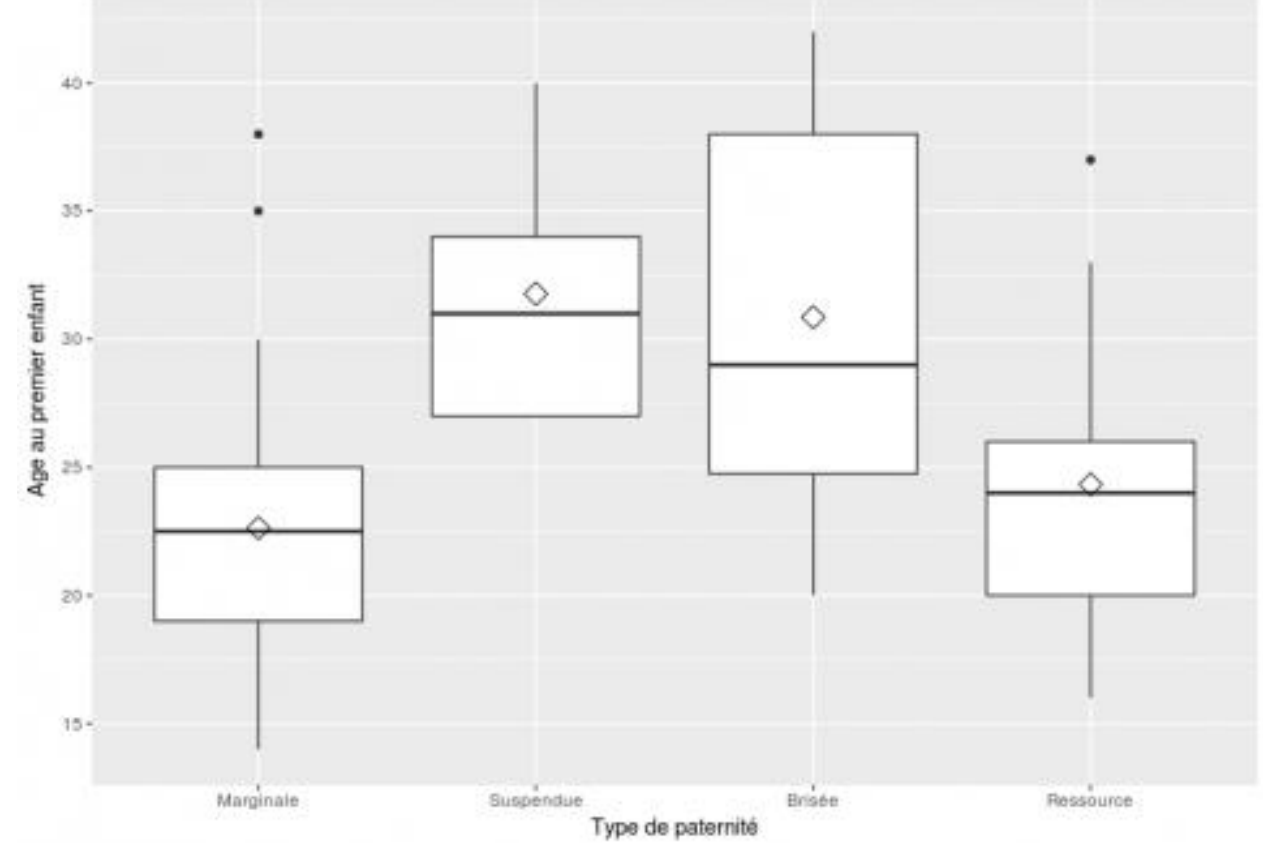

$\underline{\text { Agrandir Original (jpeg, 56k) }}$ 


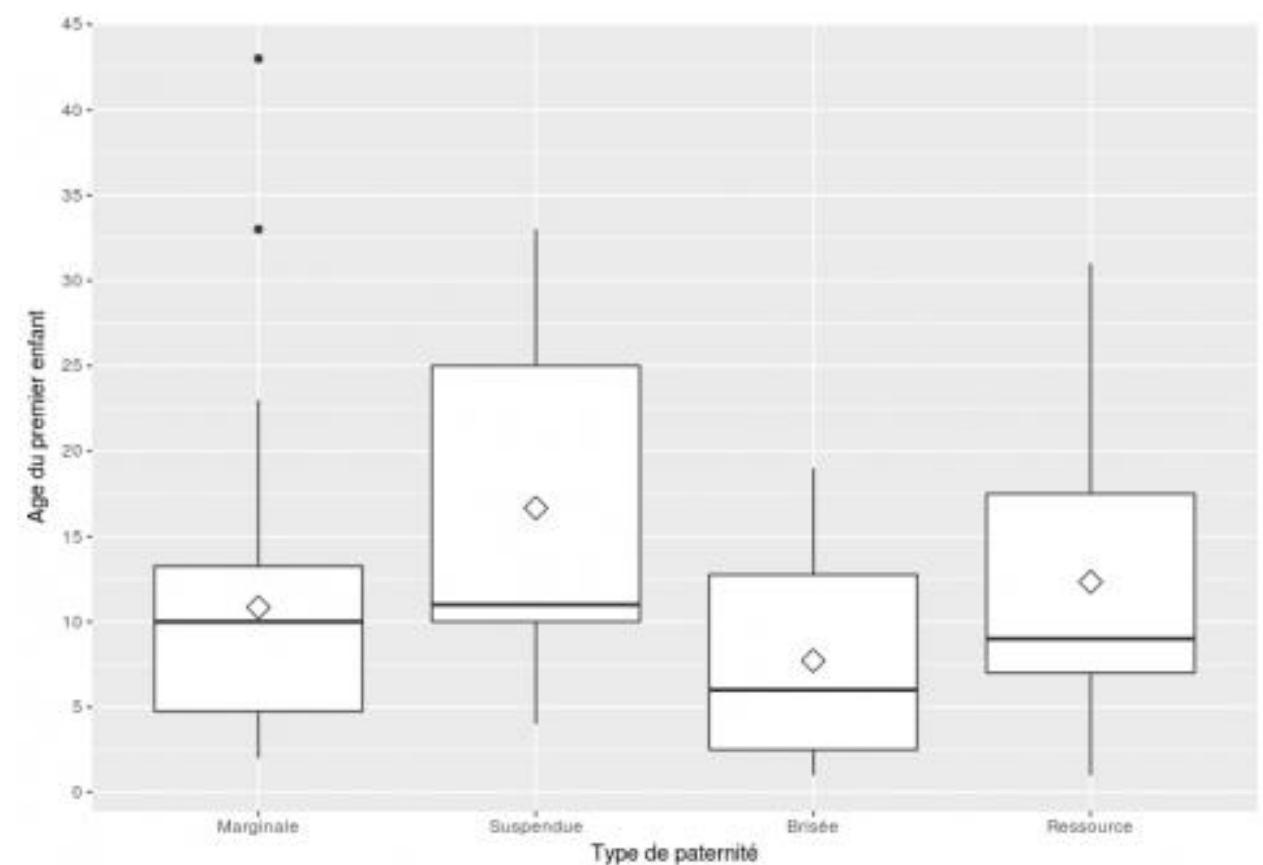

$\underline{\text { Agrandir }}$ Original (jpeg, 68k)

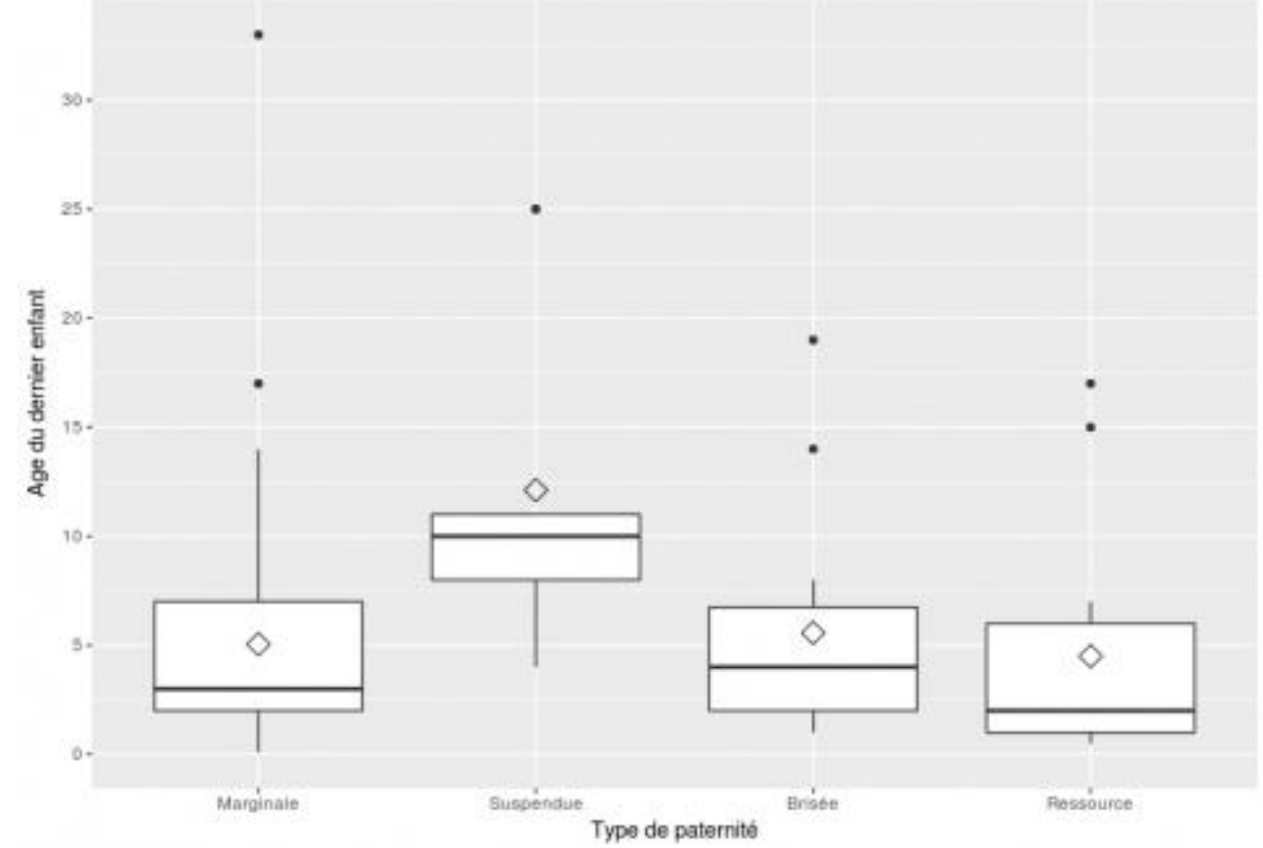

Agrandir Original (jpeg, 60k)

$\underline{\text { Haut de page }}$

\section{Notes}

1 La circulaire du 20 février 2012 relative au maintien des liens extérieurs des personnes détenues par les visites et l'envoi ou la réception d'objets précise que « suivant les termes des règles pénitentiaires européennes (règles 24.4 et 24.5), « les modalités des visites doivent permettre aux détenus de maintenir et de développer des relations familiales de façon aussi normale que possible » et « les autorités pénitentiaires doivent aider les détenus à maintenir un contact adéquat avec le monde extérieur et leur fournir l'assistance sociale appropriée pour ce faire ». Les visites concernent en effet l'exercice d'un droit fondamental, celui du droit à la 
vie familiale, protégé par l'article 8 de la Convention européenne de sauvegarde des droits de l'homme et des libertés fondamentales.

2 En Amérique du Nord, ces questions ont été abordées dès les années 1960 en termes de crise familiale, de «prisonization » (socialisation carcérale) et dans une perspective de réinsertion et de prévention de la récidive (Justin Dyer et al., 2012).

$\underline{3}$ Comme le soulignent Marie Douris et Pascal Roman $(2014,84)$, le livret reçu en début d'incarcération ne mentionne pas les droits parentaux. L'institution carcérale invisibilise cette question et ne prend pas en compte les différences sociodémographiques de la population carcérale.

4 Si un individu commet un inceste, une violence envers l'enfant ou tue l'autre parent, il est déchu de ses droits parentaux et une décision judiciaire impose l'éloignement géographique. Sans présumer l'absence d'expérience paternelle pour ce type de détenus, j'ai préféré les exclure pour éviter les difficultés spécifiques à leur situation.

$\underline{5}$ Dans mon courrier, j'expliquais mon souhait d'échanger avec eux sur leur relation à leur(s) enfant(s), leur famille, leurs amis mais aussi plus généralement sur leur parcours de vie (emploi, logement, etc.).

$\underline{6}$ Avant chaque nouvel entretien, je lisais le(s) précédent(s) entretien(s) pour avoir en mémoire les différents événements biographiques, leurs enchainements, mais également les prénoms des enfants et des compagnes. Cette préparation était fastidieuse, car je préparais pas moins de cinq ou six entretiens ne sachant pas à l'avance quel enquêté serait disponible. Je rencontrais trois détenus par jour pour optimiser mon temps sur place m'obligeant à faire une vraie gymnastique entre chaque histoire de vie. Certains enquêtés étaient exigeants quant à ma connaissance de leur histoire. Je me rappelle deux entretiens où le premier me signifiait lui avoir déjà posé les mêmes questions «mais on n'avance pas là, tout ça tu le sais déjà ». Le second me questionnait précisément sur des événements de sa vie : "alors tu te rappelles mon pays d'origine ? », « tu te rappelles ce qui m'a sauvé en prison ? ». J'ai toutefois noté le plaisir des enquêtés quand je me rappelais l'âge et le prénom de chaque enfant.

7 En master, j'ai utilisé le « vous » lors du premier entretien mais j'ai proposé rapidement aux enquêtés d'utiliser un tutoiement réciproque. En thèse, au début de chaque entretien je proposais cette forme d'échange. J'ai maintenu le vouvoiement avec quelques rares détenus plus âgés. Mon âge souvent proche de celui des enquêtés m'a conduit à adopter ce choix méthodologique présumant amoindrir la distance et favoriser un rapport de confiance. Cependant un certain nombre de détenus oscillaient entre le « vous » et le « tu » notamment lors du premier entretien.

$\underline{8}$ Je me suis rendue plusieurs fois au parloir pour réaliser des entretiens avec les surveillants. J'ai participé aux tours de parloirs (arrivée des détenus vers le parloir et départ vers la détention). Pour observer les pratiques, il aurait fallu se poster devant les cabines et y regarder par la petite fenêtre. Les surveillants n'effectuaient pratiquement plus de rondes pour préserver l'intimité des visités-visiteurs et sous peine de remontrances de leur hiérarchie et des détenus.

$\underline{9}$ Récapitulatif des caractéristiques de chacun des types en Annexe ainsi que des boxplots qui permettent de voir la médiane et la dispersion dans chacun des types pour : l'âge des détenus, 
l'âge au premier enfant, l'âge du premier et du dernier enfant ainsi que le temps passé en prison au moment du dernier entretien.

$\underline{10}$ Comme le décrivent Marie Douris et Pascal Roman $(2014,108)$, j'ai pu faire le constat que l'exercice de la paternité dépend « étroitement de la relation entretenue avec l'autre parent. » Il faut savoir que dans $71 \%$ des cas les enfants sont pris en charge par l'autre parent. Par soucis de clarté, j'ai choisi de mettre davantage l'accent sur la relation conjugale que familiale même si l'entourage familial a une part d'influence sur l'exercice de la paternité et les modèles familiaux de référence.

11 L'UVF est un appartement meublé de deux ou trois pièces, séparé de la détention, où la personne détenue peut recevoir sa famille dans l'intimité (Rambourg, 2006). En 2015, seules 36 prisons sur 188 sont dotées de tels locaux (OIP, 2016). Certains de mes enquêtés ont bénéficié de ce dispositif, mais ils ont été peu nombreux, c'est pourquoi je ne parlerai pas spécifiquement de ce dispositif dans cet article.

12 Selon l'étude sur la Précocité et instabilité familiales des hommes détenus (Cassan, MaryPortas, 2002), ce qui caractérise les détenus est « la précocité de leurs engagements familiaux et plus généralement de leurs parcours d'entrée dans la vie adulte ».

13 Âge du dernier et du premier enfant.

$\underline{14}$ Se référer pour chacun des types aux bloxplots qui permettent de voir les dispersions.

$\underline{15}$ Seuls deux détenus sur les 10 n'étant plus en couple voient de manière irrégulière leurs enfants grâce à l'ex-compagne ou à la famille paternelle.

16 Âge du dernier et du premier enfant et temps d'incarcération lors du dernier entretien.

17 Dans l'ouvrage collectif sur les sorties de délinquances (Mohammed et al., 2012).

Vaughan montre que «Les conditions matérielles, et surtout le besoin d'argent, se liguent parfois contre les délinquants qui veulent "revenir dans le droit chemin". Revêtir l'habit de la paternité peut éventuellement dissuader un délinquant de commettre des délits, mais le besoin des sortants de "nourrir leur famille" peut expliquer que certains persistent alors dans la délinquance malgré les meilleures intentions. »

$\underline{18}$ Bilal explique qu'il ne trafique jamais en présence de sa mère, sûrement pour ne pas la mêler à son mode et de vie. Pour compenser ce manque à gagner, après le parloir il propose à d'autres détenus de remonter divers produits en échange d'argent.

19 Marie Douris et Pascal Roman (2014) font le même constat dans leur étude : si «l'incarcération ne modifie pas le délitement du lien parent-enfant quand cette distance est préalable à la détention [...] elle ne l'améliore pas ».

20 Dans l'étude de Marie Douris et Pascal Roman $(2014,66)$, on constate que « 69,7\% enfants âgés de 1 à 2 ans des trois établissements pénitentiaires voient leur parent alors qu'ils ne sont que 47,3\% des enfants âgés de 11 à 14 ans pour les trois établissements et 56,4\% des enfants âgés de 15 à 17 ans ». On peut faire l'hypothèse que, pour les enfants plus âgés, leur vie quotidienne (activités), la fatigue de venir en prison et le déroulement des parloirs peuvent 
influer sur la raréfaction des visites. Concernant Bilal, son fils a 12 ans, il est donc davantage en mesure de refuser les visites.

21 Arnaud Régnier-Loilier $(2013,73)$ indique que «plus la séparation est ancienne et plus la proportion d'enfants ne voyant jamais leur père augmente (elle est de l'ordre de $3 \%$ si la séparation s'est produite un an plus tôt ou moins, $15 \%$ si elle remonte à une dizaine d'années, $25 \%$ après une vingtaine d'années). L'âge de l'enfant au moment de la séparation joue également un rôle important : plus d'un quart des enfants âgés de moins d'un an au moment de la séparation ne voient jamais leur père, contre $12 \% \mathrm{~s}$ 'ils avaient une dizaine d'années ».

22 L'étude d'Aline Désesquelles et Annie Kensey $(2006,66)$ sur les liens familiaux confirme une corrélation entre rencontres physiques et contacts indirects : « la fréquence des visites et l'intensité des échanges téléphoniques et épistolaires vont de pair ; autrement dit, il n'existe pas de phénomène de "compensation", bien au contraire. Les détenus qui ont au moins un visiteur par semaine ou par mois sont aussi plus souvent en contact téléphonique ou épistolaire avec une personne de leur famille à un rythme hebdomadaire ».

$\underline{23}$ Gwénola Ricordeau $(2008,66)$ montre que certains détenus craignent d'être rejetés et, pour conserver une image positive d'eux, refusent que les proches viennent au parloir. Elle décrit également les stratégies de dissimulation de l'incarcération.

24 Par stigmatisation je me réfère à la définition d'Erving Goffman (1970) qui décrit ce processus comme excluant des cercles sociaux les personnes du fait qu'un attribut jette le discrédit sur elles.

$\underline{25}$ Ce type de paternité partage des points communs avec « la criminelle conforme » de Coline Cardi. L'incarcération est vécue comme « un accident biographique », les enquêtées décrivant par ailleurs leur « relative stabilité professionnelle et/ou familiale » (Cardi, 2008, 279-294). Quant à Gilles Chantraine $(2004,15)$, il parle d'une « normalité sociale brisée par un crime supposé et sa répression ».

$\underline{26}$ De nombreux $\cdot$ ses auteur·e·s américain·e·s mettent en évidence deux raisons principales qui expliquent l'importance des mères dans le maintien des relations du père avec son enfant : elles sont les gardiennes des interactions et ce sont elles qui imposent ou non une restriction des visites. Le terme donné à cette restriction de l'investissement paternel par la mère est le maternal gatekeeping. Si le Relais enfant-parent peut prendre en charge l'enfant pour l'emmener en prison, les délais sont rallongés quand les mères refusent le contact (Arditti et al., 2005 ; Justin Dyer, 2005 ; Hairston, 1998 ; Nurse, 2002).

$\underline{27}$ Cette mise en récit de la «vie ordinaire » se retrouve chez la « criminelle conforme » de Cardi $(2008,300)$, cela marque « le passage d'un statut de normalité sociale [...] à un statut "d'infâme" », terme qu'elle empreinte à Chantraine $(2004,15)$

$\underline{28}$ Marie Douris et Pascal Roman $(2014,141)$ expliquent que la situation de détention provoque l'inversion « des places du point de vue de l'expérience de la parentalité ».

29 Laurent explique les difficultés de son fils à l'école dues à des troubles du comportement, qui justifieraient sa plus grande attention. 
$\underline{30}$ Les relations entre les détenus et leurs parents sont fragiles. Les enquêtés décrivent souvent des relations distantes, voire inexistantes, avec leur père. Ce dernier apparaît dans des épisodes de violences et d'alcoolisation. Si l'on s'appuie sur quelques statistiques, "plus d'un détenu sur dix ne déclare pas la profession du père, ce qui peut signifier qu'il ne l'a pas connu. Le départ du domicile parental a souvent été précoce »(Cassan, Toulemon, 2000). Les détenus revendiquent le désir d'être différents du modèle parental hérité, valorisant la présence auprès des enfants, l'écoute et la capacité à subvenir à leurs besoins. L'incarcération vient leur rappeler qu'ils ont failli par rapport à l'idéal parental qu'ils s'étaient imaginé.

$\underline{31}$ Les deux pères rencontrés qui bénéficient des Relais enfants-parents sont dans la paternité ressource. Les bénévoles de l'association ont pour mission d'accompagner l'enfant en visite auprès du parent incarcéré « afin qu'aucune circonstance extérieure (manque de temps, conflit entre les parents, enfants placés, etc.) n'entrave le maintien du lien »: http://www.relaisenfantsparents.be/missions.php.

$\underline{32}$ L'usage du téléphone et du courrier devient central quand l'enfant est en mesure de l'utiliser et surtout vers l'adolescence.

33 Marc Bessin et al. $(2009,282)$ expliquent également que pour bifurquer de manière positive, quatre éléments sont nécessaires : «la confiance en soi, le réarmement, la quiétude environnementale et la crédibilité envers les autres ».

$\underline{34}$ «L'importance de l'événement est associée au regard subjectif que cet événement provoque pour les acteurs [...] l'événement donne le sens, la direction de l'action » (Bessin et $a l ., 2009,182)$. La bifurcation se réalise dans la mise en action, dans le cas de Timur, elle est au stade de mise en récit.

$\underline{35}$ Quelques détenus refusent la « gamelle » servie par l'administration pénitentiaire, dénonçant la mauvaise qualité de la nourriture. Ils peuvent cantiner pour avoir accès à d'autres produits. Timur préfère manger la « gamelle » pour économiser de l'argent.

36 Dans l'ouvrage de Mohammed et al. (2012), de nombreux chercheurs ont montré que le choix et la décision d'abandonner des pratiques délictueuses sont la première étape de la sortie de la délinquance.

$\underline{37}$ Les détenus de ce type travaillent en détention ou tentent d'avoir un emploi et des activités (scolaires, sportives). Pour la plupart, ils cumulent des ressources (relationnelle, professionnelle, intellectuelle), il n'est donc pas étonnant qu'une partie des enquêtés soient issus des classes moyennes-supérieures.

\section{Haut de page}

\section{Table des illustrations}

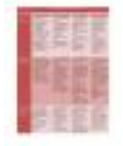

URL $\underline{\text { http://journals.openedition.org/champpenal/docannexe/image/10397/img- }}$

Fichier image/jpeg, 724k 
URL http://journals.openedition.org/champpenal/docannexe/image/10397/img2.jpg

Fichier image/jpeg, 56k

URL http://journals.openedition.org/champpenal/docannexe/image/10397/img3.jpg

Fichier image/jpeg, 56k

URL http://journals.openedition.org/champpenal/docannexe/image/10397/img4.jpg

Fichier image/jpeg, 68k

URL http://journals.openedition.org/champpenal/docannexe/image/10397/img5.jpg

Fichier image/jpeg, 60k

$\underline{\text { Haut de page }}$

\section{Pour citer cet article}

\section{Référence électronique}

Marine Quennehen, «Expérience carcérale et exercice de la paternité : le point de vue de pères détenus », Champ pénal/Penal field [En ligne], 16 | 2019, mis en ligne le 26 mars 2019, consulté le 27 mai 2019. URL : http://journals.openedition.org/champpenal/10397 ; DOI :

10.4000/champpenal.10397

$\underline{\text { Haut de page }}$

\section{Auteur}

\section{Marine Quennehen}

Institut national d'études démographiques (Ined), 75020 Paris

École des hautes études en sciences sociales (EHESS-Cespra), 75006 Paris

marine.quennehen@ined.fr

$\underline{\text { Haut de page }}$

\section{Droits d'auteur}

(C) Champ pénal 